\title{
On the existence of certain axisymmetric interior metrics
}

\author{
C. Angulo Santacruz ${ }^{1}$, D. Batic ${ }^{1,2}$ and M. Nowakowski ${ }^{3}$ \\ 1 Departamento de Matematicas, Universidad de los Andes, \\ Cra.1E No.18A-10, Bogota, Colombia \\ 2 Department of Mathematics, University of West Indies, Kingston 6, Jamaica \\ 3 Departamento de Fisica, Universidad de los Andes, \\ Cra.1E No.18A-10, Bogota, Colombia
}

\begin{abstract}
One of the effects of noncommutative coordinate operators is that the delta-function connected to the quantum mechanical amplitude between states sharp to the position operator gets smeared by a Gaussian distribution. Although this is not the full account of effects of noncommutativity, this effect is in particular important, as it removes the point singularities of Schwarzschild and ReissnerNordström solutions. In this context, it seems to be of some importance to probe also into ring-like singularities which appear in the Kerr case. In particular, starting with an anisotropic energymomentum tensor and a general axisymmetric ansatz of the metric together with an arbitrary mass distribution (e.g. Gaussian) we derive the full set of Einstein equations that the Noncommutative Geometry inspired Kerr solution should satisfy. Using these equations we prove two theorems regarding the existence of certain Kerr metrics inspired by Noncommutative Geometry.
\end{abstract}




\section{INTRODUCTION}

Noncommutative Geometry [1] has been an active field of research in the last decades. In particular, there have been several attempts [2, 3] to cure the appearance of infinities in Quantum Field Theory by means of noncommutative field theories where the formulation is done in terms of ordinary functions of commuting variables endowed with a Moyal or *-product. However, in the formulation of Noncommutative Geometry based on the aforementioned product the basic property of the noncommutativity, namely the existence of a natural ultraviolet cutoff due to the uncertainty in position, is not transparent. In particular, the free propagator is unaffected by the *-product as if noncommutativity had no effect on it. Calculations are than performed using truncated series expansion in the parameter characterizing the noncommutativity of space. This approach leads to the ultraviolet/infrared mixing phenomenon but one is still faced with divergences [4] to be cured as in ordinary Quantum Field Theory. At the present stage, we cannot exclude that the complete summation of the *-product expansion might give a finite result but such a procedure presents two major difficulties: it is far from being easy and goes beyond calculational capabilities. Therefore, even if the use of the Moyal product is a well defined procedure, its application as for now does not produce ultraviolet finiteness of Noncommutative Field Theory. On the other hand, a new formulation of Quantum Field Theory on the noncommutative plane [5] indicated that the existence of a minimal length in a noncommutative plane manifests itself already in the free propagator. The core ingredients in the formulation of this model without $*$-product is the use of expectation values of operators between coherent states [6], whereas the noncommutativity of space-time is encoded in the commutator $\left[\mathbf{x}^{\mu}, \mathbf{x}^{\nu}\right]=i \Theta^{\mu \nu}$, where $\Theta^{\mu \nu}$ is an anti-symmetric matrix which determines the fundamental cell discretization of space-time. We recall that a similar commutation relation has been already introduced in the seminal paper of Snyder [7]. In this approach, noncommutativity of coordinates is carried on by the Gaussian spread of coherent states and modifies the structure of the Feynman propagator rendering Quantum Field Theory ultraviolet finite [5]. To be more specific, the amplitude $\langle\mathbf{z} \mid \mathbf{x}\rangle$ which in the standard quantum mechanical prescription is proportional to the Dirac delta function $\delta^{(3)}(\mathbf{z}-\mathbf{x})$ can be replaced in Noncommutative Geometry by a Gaussian distribution proportional to $\exp \left(-(\mathbf{z}-\mathbf{x})^{2} / 2 \theta\right)$, where $\theta$ is a tiny parameter encoding the noncommutativity of the space-time fabric [8]. Here, it must be emphasized 
that such a replacement is also possible when one considers the Voros product instead of the Moyal product. For such a discussion, we refer to [9].

One might suspect that noncommutativity might also cure divergences appearing in various forms in General Relativity. This conjecture has been studied in [9 15], where the noncommutative inspired counterparts of the Schwarzschild and Reissner-Nordström metrics have been derived. All these new black hole geometries contain their respective classical solutions in the limit of a vanishing noncommutativity parameter $\theta$. Moreover, the central singularity is now replaced by a regular region represented by a self-gravitating, droplet of anisotropic fluid. The main idea behind the derivation of these solutions does not rely on a modification of the four-dimensional Einstein action to incorporate noncommutative effects but it consists in implementing noncommutativity only in the matter source [14]. In the present work we specialize to the axisymmetric case of the Kerr black hole where one would expect that the classical ring singularity should be replaced by a certain Gaussian distribution given in appendix D. In particular, we present the full set of Einstein equations with anisotropic energy-momentum tensor. The resulting set of equations is a highly complicated system of nonlinear partial differential equations, for which at present we do not have a solution. We recall however, that this is also the case for a similar set of equations where in contrast to us the energy-momentum tensor is isotropic [16]. The usefulness of our equations lies in the fact that any noncommutative Kerr candidate, be it an educated guess or a particular solution, must satisfy the set of equations (12)-(19). In this respect, we mention the work of [17] where the authors claimed to have derived the noncommutative counterpart of the Kerr metric. Despite the educated guess leading to the so-called Kerrr metric the authors did not perform any consistency check regarding the question whether their solution satisfies all Einstein field equations coupled to an anisotropic source or not. Here, we offer the solution to this problem presented in form of two theorems. In appendix B we performed the consistency check for the same choice of the energy-momentum tensor done in [17] and we showed that the metric represented by equation (44) in [17] can never satisfy all Einstein field equations. 


\section{THE EINSTEIN FIELD EQUATIONS WITH ANISOTROPIC MATTER SOURCE}

According to [18] the most general ansatz for metrics describing stationary axisymmetric space-times in Boyer-Lindquist coordinates $(t, R, \vartheta, \varphi)$ can be written in the form

$$
d s^{2}=\left(e^{2 \nu}-\omega^{2} e^{2 \psi}\right) d t^{2}+2 \omega e^{2 \psi} d t d \varphi-e^{2 \mu_{R}} d R^{2}-e^{2 \mu_{\vartheta}} d \vartheta-e^{2 \psi} d \varphi^{2}
$$

where $\nu, \psi, \mu_{R}$ and $\mu_{\vartheta}$ are in general functions of the spheroidal coordinates $R$ and $\vartheta$ defined through the relations [19]

$$
\frac{x^{2}+y^{2}}{R^{2}+a^{2}}+\frac{z^{2}}{R^{2}}=1, \quad \frac{x^{2}+y^{2}}{a^{2} \sin ^{2} \vartheta}-\frac{z^{2}}{a^{2} \cos ^{2} \vartheta}=1,
$$

where $a$ is a real parameter. Furthermore, we consider the Einstein field equations

$$
G_{\mu \nu}=-8 \pi T_{\mu \nu}
$$

where the energy-momentum tensor $T_{\mu \nu}$ describes an anisotropic perfect fluid with timelike four velocity $u_{\mu}$, mass density $\rho$ and pressures $p_{R}, p_{\vartheta}$. We shall derive the equations governing any stationary, axial-symmetric solution of Einstein equations (2) in the presence of an anisotropic perfect fluid, where the mass density $\rho$ is assigned from the very beginning in the spirit of $\left[10\right.$ 14, 17]. More precisely, $T_{\mu \nu}$ reads $(c=G=1$ units $)$

$$
T_{\mu \nu}=\left(\rho+p_{\vartheta}\right) u_{\mu} u_{\nu}-p_{\vartheta} g_{\mu \nu}+\left(p_{R}-p_{\vartheta}\right) \ell_{\mu} \ell_{\nu}
$$

where $\ell^{\mu}$ is a unit space-like vector orthogonal to the fluid four-velocity $u^{\mu}$, i.e.

$$
\ell^{\mu} \ell_{\mu}=-1, \quad \ell^{\mu} u_{\mu}=0
$$

Moreover, the fluid four-velocity has to satisfy the condition

$$
g_{\mu \nu} u^{\mu} u^{\nu}=1
$$

At this point a couple of remarks are in order. First of all, notice that our choice of $T_{\mu \nu}$ contains as a special case the energy-momentum tensor given by equation (37) in [17], since it reduces to $(37)$ by taking $p_{R}=-\rho$. However, in an axial-symmetric space-time there is no a priori reason to believe that the fluid should satisfy an equation of state of the de Sitter form as it is indeed the case for the spherically symmetric noncommutative geometry 
inspired Schwarzschild and Reissner-Nordström metrics. Furthermore, the choice of the fluid velocities (equation (38) in [17]) does not satisfy condition (41) with $g_{\mu \nu}$ given by the Kerrr line element (44) in [17]. In fact, a simple computation shows that

$$
g_{\mu \nu} u^{\mu} u^{\nu}=\left(u^{t}\right)^{2}\left(g_{t t}+2 \omega g_{t \varphi}+\omega^{2} g_{\varphi \varphi}\right)=\frac{\Delta^{2}}{\left(R^{2}+a^{2}\right)^{2}} \neq 1
$$

where

$$
u^{t}=\sqrt{\frac{\Delta}{\Sigma}}, \quad \omega(R)=\frac{a}{R^{2}+a^{2}}, \quad \Sigma=R^{2}+a^{2} \cos ^{2} \vartheta
$$

and $\Delta$ given by equation (45) in [17]. According to (44), the right choice of $u^{t}$ in [17] should read

$$
u^{t}=\frac{R^{2}+a^{2}}{\sqrt{\Sigma \Delta}}
$$

but even with this choice we could prove in appendix B that the so-called Kerrr metric does not satisfy all Einstein field equations.

In the present case we specialize the energy-momentum tensor to a stationary matter distribution with cylindrical symmetry. Hence, there exist two Killing vector fields $\partial_{t}$ and $\partial_{\varphi}$ and in general, there will be at least two non-vanishing velocity components $u^{t}$ and $u^{\varphi}$, so that the four velocity $u^{\mu}$ of the fluid can be written as [20]

$$
u^{\mu}=u^{t}\left(\delta_{t}^{\mu}+\Omega \delta_{\varphi}^{\mu}\right), \quad \Omega=\frac{u^{\varphi}}{u^{t}} .
$$

Here, $\Omega$ denotes the angular velocity of the fluid and depends in general on the spheroidal variables $R$ and $\vartheta$. Notice that the requirement $u^{R}=u^{\vartheta}=0$ ensures that the fluid is circulating within the $(t, \varphi)$ - surface. Condition (44) permits to express the $u^{t}$ component of the fluid velocity as

$$
u^{t}=\frac{1}{\sqrt{g_{t t}+2 \Omega g_{t \varphi}+\Omega^{2} g_{\varphi \varphi}}}=V^{-1}, \quad V=V(R, \vartheta)=\sqrt{e^{2 \nu}-(\omega-\Omega)^{2} e^{2 \psi}}
$$

whereas the non-vanishing covariant components of the four velocity are

$$
u_{t}=V^{-1}\left[e^{2 \nu}-\omega(\omega-\Omega) e^{2 \psi}\right], \quad u_{\varphi}=V^{-1}(\omega-\Omega) e^{2 \psi}
$$

Concerning the choice of the unit space-like vector $\ell^{\mu}$, we observe that it will be orthogonal to the fluid four velocity if we take $\ell^{t}=\ell^{\varphi}=0$. Moreover, the condition that $\ell^{\mu}$ be a unit space-like vector requires that

$$
e^{2 \mu_{R}}\left(\ell^{R}\right)^{2}+e^{2 \mu_{\vartheta}}\left(\ell^{\vartheta}\right)^{2}=1
$$


Notice that the above equation does not uniquely fix $\ell^{\mu}$ and we can follow at least two different approaches in determining the components of $\ell^{\mu}$. The first approach is standard and gives rise to the choice

$$
\ell^{\mu}=e^{-\mu_{R}} \delta_{R}^{\mu}, \quad \ell^{\vartheta}=0
$$

whereas the second one is represented by the more sophisticated expression

$$
\ell^{\mu}=\frac{1}{\sqrt{2}}\left(e^{-\mu_{R}} \delta_{R}^{\mu}+e^{-\mu_{\vartheta}} \delta_{\vartheta}^{\mu}\right)
$$

In order to derive the full set of Einstein field equations corresponding to each of the possible choices (5) and (6) , it is convenient to start with the following equivalent form of (2), namely

$$
R_{\mu \nu}=8 \pi\left(\frac{T}{2} g_{\mu \nu}-T_{\mu \nu}\right), \quad T=g^{\mu \nu} T_{\mu \nu}
$$

together with the conservation equation

$$
T_{; \nu}^{\mu \nu}=0
$$

However, in the coordinate approach the components of the Ricci tensor assume a very complicated form. For this reason, we shall follow the line of reasoning in [16, 18] and introduce the tetrad-frame

$$
e_{(t)}=e^{-\nu} \partial_{t}+\omega e^{-\nu} \partial_{\varphi}, \quad e_{(\varphi)}=e^{-\psi} \partial_{\varphi}, \quad e_{(R)}=e^{-\mu_{R}} \partial_{R}, \quad e_{(\vartheta)}=e^{-\mu_{\vartheta}} \partial_{\vartheta} .
$$

In this setting the relevant Einstein field equations for our problem are

$$
\begin{gathered}
R_{(t)(t)}=8 \pi\left(\frac{T}{2}-T_{(t)(t)}\right), \quad R_{(t)(\varphi)}=-8 \pi T_{(t)(\varphi)}, \\
R_{(\varphi)(\varphi)}=-8 \pi\left(\frac{T}{2}+T_{(\varphi)(\varphi)}\right), \quad G_{(R)(R)}=-8 \pi T_{(R)(R)}, \\
R_{(R)(\vartheta)}=-8 \pi T_{(R)(\vartheta)}, \quad G_{(\vartheta)(\vartheta)}=-8 \pi T_{(\vartheta)(\vartheta)} .
\end{gathered}
$$

Notice that the vanishing of the component $T_{(R)(\vartheta)}$ of the energy-momentum tensor depends on the particular choice of the unit space-like vector $\ell^{\mu}$, whereas the trace of $T^{\mu \nu}$ is for both choices of $\ell^{\mu}$ given by

$$
T=g_{\mu \nu} T^{\mu \nu}=\rho-p_{R}-2 p_{\vartheta} .
$$




\section{EINSTEIN FIELD EQUATIONS WITH $\ell^{t}=\ell^{\varphi}=\ell^{\vartheta}=0$}

The non-vanishing contravariant components of the energy-momentum tensor in the coordinate representation are

$$
\begin{gathered}
T^{t t}=V^{-2}\left[\rho+(\omega-\Omega)^{2} e^{2 \psi-2 \nu} p_{\vartheta}\right], \\
T^{t \varphi}=V^{-2}\left\{\Omega \rho-(\omega-\Omega)\left[1-\omega(\omega-\Omega) e^{2 \psi-2 \nu}\right] p_{\vartheta}\right\}, \\
T^{\varphi \varphi}=V^{-2}\left\{\Omega^{2} \rho+\left[e^{\nu-\psi}-\omega(\omega-\Omega) e^{\psi-\nu}\right]^{2} p_{\vartheta}\right\}, \quad T^{R R}=e^{-2 \mu_{R}} p_{R}, \quad T^{\vartheta \vartheta}=e^{-2 \mu_{\vartheta}} p_{\vartheta} .
\end{gathered}
$$

Taking into account that the contravariant components of the energy-momentum tensor in the non-coordinate basis can be computed from the relation $T^{(a)(b)}=e^{(a)}{ }_{\mu} e^{(b)}{ }_{\nu} T^{\mu \nu}$ with $e^{(a)}{ }_{\mu}$ defined in VIIIC, we find

$$
\begin{gathered}
T^{(t)(t)}=V^{-2}\left[e^{2 \nu} \rho+(\omega-\Omega)^{2} e^{2 \psi} p_{\vartheta}\right], \quad T^{(t)(\varphi)}=-V^{-2}(\omega-\Omega) e^{\psi+\nu}\left(\rho+p_{\vartheta}\right), \\
T^{(\varphi)(\varphi)}=V^{-2}\left[(\omega-\Omega)^{2} e^{2 \psi} \rho+e^{2 \nu} p_{\vartheta}\right], \quad T^{(R)(R)}=p_{R}, \quad T^{(\vartheta)(\vartheta)}=p_{\vartheta},
\end{gathered}
$$

whereas the covariant components are computed according to $T_{(a)(b)}=\eta_{(a)(c)} \eta_{(b)(d)} T^{(c)(d)}$. Since $\eta_{(a)(b)}=\operatorname{diag}(1,-1,-1,-1)$ (see VIIIC), we shall have $T_{(a)(b)}=T^{(a)(b)}$, whenever the tetrad indices $a$ and $b$ coincide. Moreover, if $a \neq b$, the only non zero component of the energy-momentum tensor is that with $a=t$ and $b=\varphi$ and we get $T_{(t)(\varphi)}=-T^{(t)(\varphi)}$. Finally, by means of (61)-(66) the Einstein field equations (9)-(11) can be explicitly written as

$$
\begin{aligned}
& e^{-2 \mu_{R}}\left[\nu_{, R, R}+\nu_{, R}\left(\psi+\nu-\mu_{R}+\mu_{\vartheta}\right)_{, R}\right]+e^{-2 \mu_{\vartheta}}\left[\nu_{, \vartheta, \vartheta}+\nu_{, \vartheta}\left(\psi+\nu+\mu_{R}-\mu_{\vartheta}\right)_{, \vartheta}\right]-\frac{1}{2} e^{2(\psi-\nu)} . \\
& {\left[\left(\omega_{, R}\right)^{2} e^{-2 \mu_{R}}+\left(\omega_{, \vartheta}\right)^{2} e^{-2 \mu_{\vartheta}}\right]=-\frac{4 \pi}{V^{2}}\left[e^{2 \nu}\left(\rho+p_{R}\right)+(\omega-\Omega)^{2} e^{2 \psi}\left(\rho-p_{R}\right)+2 e^{2 \nu} p_{\vartheta}\right],} \\
& \frac{1}{2} e^{-2 \psi-\mu_{R}-\mu_{\vartheta}}\left[\left(\omega_{, R} e^{3 \psi-\nu-\mu_{R}+\mu_{\vartheta}}\right)_{, R}+\left(\omega_{, \vartheta} e^{3 \psi-\nu-\mu_{\vartheta}+\mu_{R}}\right)_{, \vartheta}\right] \\
& =-\frac{8 \pi}{V^{2}}(\omega-\Omega) e^{\psi+\nu}\left(\rho+p_{\vartheta}\right) \\
& e^{-2 \mu_{R}}\left[\psi_{, R, R}+\psi_{, R}\left(\psi+\nu-\mu_{R}+\mu_{\vartheta}\right)_{, R}\right]+e^{-2 \mu_{\vartheta}}\left[\psi_{, \vartheta, \vartheta}+\psi_{, \vartheta}\left(\psi+\nu+\mu_{R}-\mu_{\vartheta}\right)_{, \vartheta}\right] \\
& +\frac{1}{2} e^{2(\psi-\nu)}\left[\left(\omega_{, R}\right)^{2} e^{-2 \mu_{R}}+\left(\omega_{, \vartheta}\right)^{2} e^{-2 \mu_{\vartheta}}\right]=4 \pi\left[\rho-p_{R}+\frac{2(\omega-\Omega)^{2} e^{2 \psi}}{V^{2}}\left(\rho+p_{\vartheta}\right)\right] \\
& (\psi+\nu)_{, R, \vartheta}-(\psi+\nu)_{, R} \mu_{R, \vartheta}-(\psi+\nu)_{, \vartheta} \mu_{\vartheta, R}+\psi_{, R} \psi_{, \vartheta}+\nu_{, R} \nu_{, \vartheta}=\frac{1}{2} \omega_{, R} \omega_{, \vartheta} e^{2(\psi-\nu)}, \\
& e^{-2 \mu_{R}}\left[\nu_{, R}\left(\psi+\mu_{\vartheta}\right)_{, R}+\psi_{, R} \mu_{\vartheta, R}\right]+e^{-2 \mu_{\vartheta}}\left[(\psi+\nu)_{, \vartheta, \vartheta}+(\psi+\nu)_{, \vartheta}\left(\nu-\mu_{\vartheta}\right)_{, \vartheta}+\psi_{, \vartheta} \psi_{, \vartheta}\right]
\end{aligned}
$$




$$
\begin{gathered}
+\frac{1}{4} e^{2 \psi-2 \nu}\left[\left(\omega_{, R}\right)^{2} e^{-2 \mu_{R}}-\left(\omega_{, \vartheta}\right)^{2} e^{-2 \mu_{\vartheta}}\right]=-8 \pi p_{R} \\
\left.\left.e^{-2 \mu_{R}\left[(\psi+\nu)_{, R, R}\right.}+(\psi+\nu)_{, R}\left(\nu-\mu_{R}\right)_{, R}+\psi_{, R} \psi_{, R}\right]+e^{-2 \mu_{\vartheta}}\left[\nu, \psi+\mu_{R}\right)_{, \vartheta}+\psi_{, \vartheta} \mu_{R, \vartheta}\right] \\
-\frac{1}{4} e^{2 \psi-2 \nu}\left[\left(\omega_{, R}\right)^{2} e^{-2 \mu_{R}}-\left(\omega_{, \vartheta}\right)^{2} e^{-2 \mu_{\vartheta}}\right]=-8 \pi p_{\vartheta}
\end{gathered}
$$

Notice that the system of six equations (12)-(17) is under-determined, since it involves the eight unknowns $\nu, \psi, \omega, \mu_{R}, \mu_{\vartheta}, p_{R}, p_{\vartheta}$ and $\Omega$. The additional two equations needed to close the system are provided by the conservation equation

$$
T_{; \nu}^{\mu \nu}=T_{, \nu}^{\mu \nu}+\Gamma_{\nu \lambda}^{\mu} T^{\lambda \nu}+\Gamma_{\nu \lambda}^{\nu} T^{\mu \lambda}=0 .
$$

For $\mu=R$ we obtain

$$
\begin{gathered}
p_{R, R}+\left(\psi+\nu+\mu_{\vartheta}\right)_{, R} p_{R}+\left[\nu_{,} e^{2 \nu}-(\omega-\Omega) \omega_{, R} e^{2 \psi}-(\omega-\Omega)^{2} \psi_{, R} e^{2 \psi}\right] \frac{\rho}{V^{2}} \\
+\left[-\left(\psi+\mu_{\vartheta}\right)_{, R} e^{2 \nu}-(\omega-\Omega) \omega_{, R} e^{2 \psi}+(\omega-\Omega)^{2}\left(\nu+\mu_{\vartheta}\right)_{, R} e^{2 \psi}\right] \frac{p_{\vartheta}}{V^{2}}=0
\end{gathered}
$$

whereas for $\mu=\vartheta$ we have

$$
\begin{gathered}
p_{\vartheta, \vartheta}+\left[\left(\nu+\mu_{R}\right)_{, \vartheta} e^{2 \nu}-(\omega-\Omega) \omega_{, \vartheta} e^{2 \psi}-(\omega-\Omega)^{2}\left(\psi+\mu_{R}\right)_{, \vartheta} e^{2 \psi}\right] \frac{p_{\vartheta}}{V^{2}} \\
+\left[\nu_{, \vartheta} e^{2 \nu}-(\omega-\Omega) \omega_{, \vartheta} e^{2 \psi}-(\omega-\Omega)^{2} \psi_{, \vartheta} e^{2 \psi}\right] \frac{\rho}{V^{2}}-\mu_{R, \vartheta} p_{R}=0 .
\end{gathered}
$$

The set of eight equations (12)-(19) for the eight unknown functions $\nu, \psi, \mu_{R}, \mu_{\vartheta}, \omega, \Omega, p_{R}$, and $p_{\vartheta}$ represents the full set of Einstein field equations assuming an anisotropic energymomentum tensor. If the energy density is inspired by Noncommutative Geometry in the spirit of [10 14, 17] and an equation of state of the de Sitter form is assumed, namely $\rho=-p_{R}$, it is not difficult to verify that equation (18) becomes

$$
-p_{\vartheta}=\rho+\frac{V^{2}}{\Phi} \rho_{, R}
$$

where

$$
\Phi=\Phi(R, \vartheta)=\left(\psi+\mu_{\vartheta}\right)_{,} e^{2 \nu}+(\omega-\Omega) \omega_{, R} e^{2 \psi}-(\omega-\Omega)^{2}\left(\nu+\mu_{\vartheta}\right)_{, R} e^{2 \psi}
$$

Equation (20) generalizes formula (41) in [17] to any stationary, axial-symmetric geometry generated by a perfect anisotropic fluid with $\ell^{\mu}$ chosen as in the present section. 


\section{EINSTEIN FIELD EQUATIONS WITH $\ell^{t}=\ell^{\varphi}=0$}

In the case of $\ell^{\mu}$ specified by relation (6) attention has to be paid to the contravariant component $T^{R \vartheta}$ of the energy-momentum tensor, since it does not vanish any longer and is now given by

$$
T^{R \vartheta}=\frac{1}{2}\left(p_{R}-p_{\vartheta}\right) e^{-\left(\mu_{R}+\mu_{\vartheta}\right)}
$$

Moreover,

$$
T^{\vartheta \vartheta}=\frac{1}{2}\left(p_{R}+p_{\vartheta}\right) e^{-2 \mu_{\vartheta}}, \quad T^{R R}=\frac{1}{2}\left(p_{R}+p_{\vartheta}\right) e^{-2 \mu_{R}},
$$

whereas the remaining non-vanishing components are the same as those given in the previous section. The corresponding contravariant components in the non-coordinate basis read

$$
T^{(R)(R)}=T^{(\vartheta)(\vartheta)}=\frac{1}{2}\left(p_{R}+p_{\vartheta}\right), \quad T^{(R)(\vartheta)}=\frac{1}{2}\left(p_{R}-p_{\vartheta}\right) .
$$

Einstein field equations (12)-(14) remain the same, whereas (15)-(17) become now

$$
\begin{aligned}
& (\psi+\nu)_{, R, \vartheta}-(\psi+\nu)_{, R} \mu_{R, \vartheta}-(\psi+\nu)_{, \vartheta} \mu_{\vartheta, R} \\
& +\psi_{, R} \psi_{, \vartheta}+\nu_{, R} \nu_{, \vartheta}-\frac{1}{2} \omega_{, R} \omega_{, \vartheta} e^{2(\psi-\nu)}=4 \pi\left(p_{R}-p_{\vartheta}\right) e^{-\left(\mu_{R}+\mu_{\vartheta}\right)}, \\
& e^{-2 \mu_{R}}\left[\nu_{, R}\left(\psi+\mu_{\vartheta}\right)_{, R}+\psi_{, R} \mu_{\vartheta, R}\right]+e^{-2 \mu_{\vartheta}}\left[(\psi+\nu)_{, \vartheta, \vartheta}+(\psi+\nu)_{, \vartheta}\left(\nu-\mu_{\vartheta}\right)_{, \vartheta}+\psi_{, \vartheta} \psi_{, \vartheta}\right] \\
& +\frac{1}{4} e^{2 \psi-2 \nu}\left[\left(\omega_{, R}\right)^{2} e^{-2 \mu_{R}}-\left(\omega_{, \vartheta}\right)^{2} e^{-2 \mu_{\vartheta}}\right]=-4 \pi\left(p_{R}+p_{\vartheta}\right), \\
& e^{-2 \mu_{R}}\left[(\psi+\nu)_{, R, R}+(\psi+\nu)_{, R}\left(\nu-\mu_{R}\right)_{, R}+\psi_{, R} \psi_{, R}\right]+e^{-2 \mu_{\vartheta}}\left[\nu_{, \vartheta}\left(\psi+\mu_{R}\right)_{, \vartheta}+\psi_{, \vartheta} \mu_{R, \vartheta}\right] \\
& -\frac{1}{4} e^{2 \psi-2 \nu}\left[\left(\omega_{, R}\right)^{2} e^{-2 \mu_{R}}-\left(\omega_{, \vartheta}\right)^{2} e^{-2 \mu_{\vartheta}}\right]=-4 \pi\left(p_{R}+p_{\vartheta}\right) .
\end{aligned}
$$

In this case the hydrodynamic equations are

$$
\begin{gathered}
\left(p_{R}+p_{\vartheta}\right)_{, R}+(\psi+\nu)_{, R}\left(p_{R}+p_{\vartheta}\right)+2\left[\nu_{, R} e^{2 \nu}-(\omega-\Omega) \omega_{, R} e^{2 \psi}-(\omega-\Omega)^{2} \psi,_{, R} e^{2 \psi}\right] \frac{\rho}{V^{2}} \\
+2\left[-\left(\psi+\mu_{\vartheta}\right)_{, R} e^{2 \nu}-(\omega-\Omega) \omega_{, R} e^{2 \psi}+(\omega-\Omega)^{2}\left(\nu+\mu_{\vartheta}\right)_{, R} e^{2 \psi}\right] \frac{p_{\vartheta}}{V^{2}} \\
+\left[\left(\nu+2 \mu_{R}+\psi\right)_{, \vartheta}\left(p_{R}-p_{\vartheta}\right)+\left(p_{R}-p_{\vartheta}\right)_{, \vartheta}\right] e^{\mu_{R}-\mu_{\vartheta}}=0
\end{gathered}
$$

whereas for $\mu=\vartheta$ we have

$$
\begin{aligned}
\left(p_{R}+p_{\vartheta}\right)_{, \vartheta}+ & (\psi+\nu)_{, \vartheta}\left(p_{R}+p_{\vartheta}\right)+2\left[\nu_{, \vartheta} e^{2 \nu}-(\omega-\Omega) \omega_{, \vartheta} e^{2 \psi}-(\omega-\Omega)^{2} \psi_{, \vartheta} e^{2 \psi}\right] \frac{\rho}{V^{2}} \\
& +2\left[-\psi_{, \vartheta} e^{2 \nu}-(\omega-\Omega) \omega_{, \vartheta} e^{2 \psi}+(\omega-\Omega)^{2} \nu_{, \vartheta} e^{2 \psi}\right] \frac{p_{\vartheta}}{V^{2}} \\
+ & {\left[\left(\nu+2 \mu_{\vartheta}+\psi\right)_{, R}\left(p_{R}-p_{\vartheta}\right)+\left(p_{R}-p_{\vartheta}\right)_{, R}\right] e^{-\mu_{R}+\mu_{\vartheta}}=0 }
\end{aligned}
$$




\section{EINSTEIN FIELD EQUATIONS FOR KERR-LIKE METRICS}

Let us suppose that the metric (1) admits an event horizon, i.e. a smooth two-dimensional null surface spanned by the Killing vectors $\partial_{t}$ and $\partial_{\varphi}$. Suppose further that the equation of this horizon be $H(R, \vartheta)=0$. Such a surface will be null if

$$
g^{\mu \nu} H_{, \mu} H_{, \nu}=0
$$

that is

$$
e^{2\left(\mu_{\vartheta}-\mu_{R}\right)}\left(H_{, R}\right)^{2}+\left(H_{, \vartheta}\right)^{2}=0 .
$$

Furthermore, the gauge freedom allows to suppose that

$$
e^{2\left(\mu_{\vartheta}-\mu_{R}\right)}=\Delta(R)
$$

and (26) implies that the equation of the event horizon is simply $\Delta(R)=0$. Moreover, the condition that the surface describing the event horizon be spanned by the Killing vectors $\partial_{t}$ and $\partial_{\varphi}$ requires that the determinant of the metric of the subspace $(t, \varphi)$ vanish on $\Delta(R)=0$, i.e. $e^{2(\psi+\nu)}=0$ on $\Delta(R)=0$. Without loss of generality we can suppose that

$$
e^{\beta}=e^{\psi+\nu}=\sqrt{\Delta} F(R, \theta)
$$

where $F$ is some function of $R$ and $\vartheta$ that we demand to be regular on $\Delta(R)=0$ and on the axis $\vartheta=0$. In virtue of the previous considerations we can establish the following lemma:

Lemma 1 Under the condition that there exists at least one horizon the metric (1) can be rewritten as

$$
d s^{2}=e^{\beta}\left(\chi-\frac{\omega^{2}}{\chi}\right) d t^{2}+2 \frac{\omega e^{\beta}}{\chi} d t d \varphi-\frac{e^{2 \mu_{\vartheta}}}{\Delta} d R^{2}-e^{2 \mu_{\vartheta}} d \vartheta^{2}-\frac{e^{\beta}}{\chi} d \varphi^{2}
$$

with $\chi=e^{-\psi+\nu}$.

Let us introduce the vierbein

$$
e^{(t)}=e^{\beta / 2} \sqrt{\chi} d t, \quad e^{\varphi}=\frac{e^{\beta / 2}}{\sqrt{\chi}}(d \varphi-\omega d t), \quad e^{(R)}=\frac{e^{\mu_{\vartheta}}}{\sqrt{\Delta}}, \quad e^{\vartheta}=e^{\mu_{\vartheta}} .
$$

We consider the energy-momentum tensor given as in (3). In the present case, the nonvanishing contravariant components of the fluid velocity are

$$
u^{t}=e^{-\beta / 2} \sqrt{\frac{\chi}{\chi^{2}-(\omega-\Omega)^{2}}}, \quad u^{\varphi}=\Omega u^{t} .
$$


with $\Omega$ depending in general on $R$ and $\vartheta$, whereas the unit space-like vector $\ell^{\mu}$ can be chosen to be

$$
\ell^{\mu}=\sqrt{\Delta} e^{-\mu_{\vartheta}} \delta_{R}^{\mu}
$$

Finally, the covariant components of the energy-momentum tensor in the non-coordinate basis introduced above are

$$
\begin{gathered}
T_{(t)(t)}=\frac{\chi^{2} \rho+(\omega-\Omega)^{2} p_{\vartheta}}{\chi^{2}-(\omega-\Omega)^{2}}, \quad T_{(t)(\varphi)}=\frac{(\omega-\Omega) \chi}{\chi^{2}-(\omega-\Omega)^{2}}\left(\rho+p_{\vartheta}\right), \\
T_{(\varphi)(\varphi)}=\frac{(\omega-\Omega)^{2} \rho+\chi^{2} p_{\vartheta}}{\chi^{2}-(\omega-\Omega)^{2}}, \quad T_{(R)(R)}=p_{R}, \quad T^{(\vartheta)(\vartheta)}=p_{\vartheta} .
\end{gathered}
$$

Following [18], it results convenient to consider Einstein field equations as taken below

$$
\begin{gathered}
R_{(t)(t)}-R_{(\varphi)(\varphi)}=8 \pi\left(T-T_{(t)(t)}+T_{(\varphi)(\varphi)}\right), \\
R_{(t)(t)}+R_{(\varphi)(\varphi)}=-8 \pi\left(T_{(t)(t)}+T_{(\varphi)(\varphi)}\right), \\
R_{(t)(\varphi)}=-8 \pi T_{(t)(\varphi)}, \\
R_{(R)(\vartheta)}=0, \\
G_{(R)(R)}-G_{(\vartheta)(\vartheta)}=-8 \pi\left(T_{(R)(R)}-T_{(\vartheta)(\vartheta)}\right) . \\
G_{(R)(R)}+G_{(\vartheta)(\vartheta)}=-8 \pi\left(T_{(R)(R)}+T_{(\vartheta)(\vartheta)}\right),
\end{gathered}
$$

The reason for this is twofold. First, it permits to express Einstein field equations in a compact form in terms of the unknown functions appearing in the metric (29). Second, when we rewrite the above equations in terms of the unknown functions $\beta, \Delta, \chi, \omega$ and $\mu_{\vartheta}$, it results that equation (35) coincides with equation (30) showing that not all Einstein field equations (30)-(35) are independent. For our present purposes it is sufficient to consider equations (30)-(34), which now read

$$
\begin{gathered}
\left(\sqrt{\Delta}(\sqrt{\Delta} F)_{, R}\right)_{, R}+F_{, \vartheta, \vartheta}=-8 \pi F e^{2 \mu_{\vartheta}}\left(p_{R}+p_{\vartheta}\right), \\
\left(\Delta F(\ln \chi)_{, R}\right)_{, R}+\left(F(\ln \chi)_{, \vartheta}\right)_{, \vartheta}-\frac{F}{\chi^{2}}\left[\Delta\left(\omega_{, R}\right)^{2}+\left(\omega_{, \vartheta}\right)^{2}\right]=-8 \pi f F e^{2 \mu_{\vartheta}}\left(\rho+p_{\vartheta}\right), \\
\left(\frac{\Delta F}{\chi^{2}} \omega_{, R}\right)_{, R}+\left(\frac{F}{\chi^{2}} \omega_{, \vartheta}\right)_{, \vartheta}=-16 \pi g F e^{2 \mu_{\vartheta}}\left(\rho+p_{\vartheta}\right), \\
(\ln F)_{, R, \vartheta}-\left[(\ln (\sqrt{\Delta} F))_{, R} \mu_{\vartheta, \vartheta}+(\ln F)_{, \vartheta} \mu_{\vartheta, R}\right]+\frac{1}{2}(\ln (\sqrt{\Delta} F))_{, R}(\ln F)_{, \vartheta}
\end{gathered}
$$




$$
\begin{gathered}
+\frac{\chi_{, R} \chi_{, \vartheta}-\omega_{, R} \omega_{, \vartheta}}{2 \chi^{2}}=0 \\
\left(\sqrt{\Delta}(\sqrt{\Delta} F)_{, R}\right)_{, R}-F_{, \vartheta, \vartheta}+2 F\left[(\ln F)_{, \vartheta}\left(\frac{1}{4}(\ln F)_{, \vartheta}+\mu_{\vartheta, \vartheta}\right)-\frac{1}{4}\left((\ln \chi)_{, \vartheta}\right)^{2}\right] \\
-2 \Delta F\left[(\ln (\sqrt{\Delta} F))_{, R}\left(\frac{1}{4}(\ln (\sqrt{\Delta} F))_{, R}+\mu_{\vartheta, R}\right)-\frac{1}{4}\left((\ln \chi)_{, R}\right)^{2}\right] \\
-\frac{F}{2 \chi^{2}}\left[\Delta\left(\omega_{, R}\right)^{2}-\left(\omega_{, \vartheta}\right)^{2}\right]=8 \pi F e^{2 \mu_{\vartheta}}\left(p_{R}-p_{\vartheta}\right)
\end{gathered}
$$

with

$$
f(\chi, \omega, \Omega)=\frac{\chi^{2}+(\omega-\Omega)^{2}}{\chi^{2}-(\omega-\Omega)^{2}}, \quad g(\chi, \omega, \Omega)=\frac{\omega-\Omega}{\chi^{2}-(\omega-\Omega)^{2}},
$$

whereas the conservation equation for the energy-momentum tensor gives rise to the following equations

$$
\begin{gathered}
p_{R, R}+\left(\beta+\mu_{\vartheta}\right)_{, R} p_{R}+\frac{f_{+} \rho+f_{-} p_{\vartheta}}{2 \chi\left[\chi^{2}-(\omega-\Omega)^{2}\right]}=0, \\
p_{\vartheta, \vartheta}-\mu_{\vartheta, \vartheta} p_{R}+\frac{g_{+} \rho+g_{-} p_{\vartheta}}{2 \chi\left[\chi^{2}-(\omega-\Omega)^{2}\right]}=0,
\end{gathered}
$$

where $\beta=\ln (\sqrt{\Delta} F)$ and

$$
\begin{gathered}
f_{+}=f_{+}(R, \vartheta)=\chi^{2}\left(\chi \beta_{, R}+\chi, R\right)-2(\omega-\Omega) \chi \omega_{, R}-(\omega-\Omega)^{2}\left(\chi \beta_{, R}-\chi_{, R}\right), \\
f_{-}=f_{-}(R, \vartheta)=-\chi^{2}\left(\chi \beta_{, R}-\chi_{, R}+2 \mu_{\vartheta, R} \chi\right)-2(\omega-\Omega) \chi \omega_{, R} \\
+(\omega-\Omega)^{2}\left(\chi \beta_{, R}+\chi_{, R}+2 \mu_{\vartheta, R} \chi\right), \\
g_{+}=g_{+}(R, \vartheta)=\chi^{2}\left(\chi \beta_{, \vartheta}+\chi_{, \vartheta}\right)-2(\omega-\Omega) \chi \omega_{, \vartheta}-(\omega-\Omega)^{2}\left(\chi \beta_{, \vartheta}-\chi_{, \vartheta}\right), \\
g_{-}=g_{-}(R, \vartheta)=\chi^{2}\left(\chi \beta_{, \vartheta}+\chi_{, \vartheta}+2 \mu_{\vartheta, \vartheta} \chi\right)-2(\omega-\Omega) \chi \omega_{, \vartheta} \\
-(\omega-\Omega)^{2}\left(\chi \beta_{, \vartheta}-\chi_{, \vartheta}+2 \mu_{\vartheta, \vartheta} \chi\right) .
\end{gathered}
$$

A this point a couple of remarks are in order. First of all, as expected equation (36) reduces to equation (43) at page 279 in [18] whenever $F$ depends only on the angular variable $\vartheta$. In this case one recovers the well known result for the Kerr metric given by $\Delta(R)=R^{2}-2 M R+a^{2}$. Furthermore, the system of five equations (36)-(40) contains 8 unknowns, namely five metric functions and the mass density, the radial pressure and the tangential pressure, which until now we left unspecified. To circumvent this problem we recall that we are interested in Noncommutative Geometry inspired Kerr-like solutions and hence, the mass density $\rho$ is an assigned function and an equation of state of the de Sitter form, namely $p_{R}=-\rho$ will be 
assumed following the line of reasoning in [10 14, 17]. Finally, the pressures $p_{\vartheta}$ and $p_{\varphi}$ can be computed by invoking the conservation equation for the energy-momentum tensor. In this setting (41) reduces as expected to the equation

$$
-p_{\vartheta}=\rho+U \rho_{, R}
$$

where

$$
U=U(R, \vartheta)=-\frac{2 \chi}{f_{-}}\left[\chi^{2}-(\omega-\Omega)^{2}\right]
$$

Finally, the next theorem shows that the metric (44) given in [17] can never satisfy Einstein Field equations (36) - (40).

Theorem 1 Under the condition that there exists at least one horizon the Einstein field equations (36)- (40) with energy-momentum tensor given by

$$
T_{\nu}^{\mu}=\left(\rho+p_{\vartheta}\right)\left(u^{\mu} u_{\nu}-\ell^{\mu} \ell_{\nu}\right)-p_{\vartheta} \delta_{\nu}^{\mu}, \quad \ell^{\mu}=e^{-\mu_{\vartheta}} \sqrt{\Delta} \delta_{R}^{\mu}, \quad \ell^{\vartheta}=0
$$

with

$$
\rho(R, \theta)=\frac{R^{4}}{\Sigma^{2}} \rho_{G}(R), \quad \Sigma=R^{2}+a^{2} \cos ^{2} \vartheta, \quad \rho_{G}(R)=\frac{M}{\left(4 \pi \ell_{0}\right)^{3 / 2}} e^{-R^{2} / 4 \ell_{0}^{2}}
$$

do not admit any solution of the form

$$
\begin{gathered}
d s^{2}=\left(1-\frac{2 R M(R)}{\Sigma}\right) d t^{2}+\frac{4 a R M(R)}{\Sigma} \sin ^{2} \vartheta d t d \varphi-\frac{\Sigma}{\Delta} d R^{2}-\Sigma d \vartheta^{2} \\
-\frac{\sin ^{2} \vartheta}{\Sigma}\left[\left(R^{2}+a^{2}\right)^{2}-a^{2} \sin ^{2} \vartheta \Delta\right] d \varphi^{2}
\end{gathered}
$$

where

$$
\Delta=R^{2}-2 R M(R)+a^{2}, \quad M(R)=4 \pi \int_{0}^{R} d x x^{2} \rho_{G}(x) .
$$

Proof. It sufficient to show that the Einstein field equation (36) will never be satisfied by the metric (45). First of all, comparing the metrics (29) and (45) we find that our function $F$ has to depend only on the angular variable $\vartheta$ and more precisely, $F(\vartheta)=\sin \vartheta$. Notice that the same choice occurs in the derivation of the classic Kerr metric [18]. Moreover, the function $\Delta$ can be identified with $\Delta=R^{2}-2 R M(R)+a^{2}$ and $e^{2 \mu_{\vartheta}}=\Sigma$. If we consider an equation of state of the form $p_{R}=-\rho$ as in [17], equation (36) simplifies as follows

$$
\frac{d^{2} \Delta}{d R^{2}}-2=16 \pi \Sigma\left(\rho-p_{\vartheta}\right)
$$


Taking into account equation (41) in [17] or equivalently our (43), the r.h.s. of (47) can be written in terms of $\rho$ and its derivative with respect to the space-like variable $R$. Thus, we find the equation

$$
\frac{d^{2} \Delta}{d R^{2}}=2+8\left(4 \pi R^{2} \rho_{G}+\pi R^{3} \frac{d \rho_{G}}{d R}\right)
$$

whose solution is

$$
\Delta(R)=R^{2}+C_{1} R+C_{2}+\frac{M}{2} \sqrt{2 \ell_{0}} R\left[\ell_{0} \operatorname{erf}\left(\frac{R}{2 \ell_{0}}\right)-\frac{R}{\sqrt{\pi}} e^{-R^{2} / 4 \ell_{0}}\right] .
$$

But $\Delta(R)$ was already given by (46) whose integration does not agree with the above expression. Therefore, we have a contradiction.

We can sharpen the above statement by noticing that alone from (46) we obtain

$$
\frac{d^{2} \Delta}{d R^{2}}=2-8\left(4 \pi R^{2} \rho_{G}+\pi R^{3} \frac{d \rho_{G}}{d R}\right),
$$

which is not the same as (48). Hence, we can assert

Corollary 1 The statement of Theorem 1 is independent of the choice of the density $\rho$.

Notice that the Theorem 1 has been proved by assuming the existence of a horizon. How-

ever, this is not necessary. Indeed, with the correct choice of the four-velocities, i.e, $u^{\mu}=\frac{R^{2}+a^{2}}{\sqrt{\Sigma \Delta}}\left(\delta_{t}^{\mu}+\Omega \delta_{\varphi}^{\mu}\right), \Omega=\frac{a}{R^{2}+a^{2}}$ we can prove the following:

Theorem 2 The Einstein field equations

$$
G_{\mu \nu}=-8 \pi T_{\mu \nu}
$$

with energy-momentum tensor given by

$$
T_{\nu}^{\mu}=\left(\rho+p_{\vartheta}\right)\left(u^{\mu} u_{\nu}-\ell^{\mu} \ell_{\nu}\right)-p_{\vartheta} \delta_{\nu}^{\mu}
$$

and

$$
u^{\mu}=\frac{R^{2}+a^{2}}{\sqrt{\Sigma \Delta}}\left(\delta_{t}^{\mu}+\Omega \delta_{\varphi}^{\mu}\right), \quad \frac{u^{\varphi}}{u^{t}}=\Omega=\frac{a}{R^{2}+a^{2}}, \quad \ell^{\mu}=-\sqrt{\frac{\Delta}{\Sigma}} \delta_{R}^{\mu}
$$

do not admit any solution of the form

$$
\begin{gathered}
d s^{2}=\left(1-\frac{2 R M(R)}{\Sigma}\right) d t^{2}+\frac{4 a R M(R)}{\Sigma} \sin ^{2} \vartheta d t d \varphi-\frac{\Sigma}{\Delta} d R^{2}-\Sigma d \vartheta^{2} \\
-\frac{\sin ^{2} \vartheta}{\Sigma}\left[\left(R^{2}+a^{2}\right)^{2}-a^{2} \sin ^{2} \vartheta \Delta\right] d \varphi^{2}
\end{gathered}
$$

with

$$
\Sigma=R^{2}+a^{2} \cos ^{2} \vartheta, \quad \Delta=R^{2}-2 R M(R)+a^{2} .
$$


Proof. We begin by studying the conservation equation $T_{; \nu}^{\mu \nu}=0$. For $\mu=R$ we obtain

$$
\partial_{R} T^{R R}+\Gamma_{t t}^{R} T^{t t}+2 \Gamma_{t \varphi}^{R} T^{t \varphi}+\Gamma_{\varphi \varphi}^{R} T^{\varphi \varphi}+\left(\Gamma_{t R}^{t}+\Gamma_{\varphi R}^{\varphi}+2 \Gamma_{R R}^{R}+\Gamma_{\vartheta R}^{\vartheta}\right) T^{R R}+\Gamma_{\vartheta \vartheta}^{R} T^{\vartheta \vartheta}=0 .
$$

Taking into account that

$$
\begin{gathered}
T^{R R}=-\frac{\Delta}{\Sigma} \rho, \quad T^{t t}=\frac{\left(R^{2}+a^{2}\right)^{2}}{\Sigma \Delta} \rho+\frac{a^{2} \sin ^{2} \vartheta}{\Sigma} p_{\vartheta}, \quad T^{t \varphi}=\frac{a\left(R^{2}+a^{2}\right)}{\Sigma \Delta} \rho+\frac{a}{\Sigma} p_{\vartheta} \\
T^{\varphi \varphi}=\frac{a^{2}}{\Sigma \Delta} \rho+\frac{1}{\Sigma \sin ^{2} \vartheta} p_{\vartheta}, \quad T^{\vartheta \vartheta}=\frac{p_{\vartheta}}{\Sigma}
\end{gathered}
$$

and

$$
\begin{gathered}
\Gamma_{t t}^{R}=-\frac{\Delta}{\Sigma^{3}}\left[R \Sigma \frac{d M}{d R}-\left(R^{2}-a^{2} \cos ^{2} \vartheta\right) M(R)\right], \quad \Gamma_{t \varphi}^{R}=-a \sin ^{2} \vartheta \Gamma_{t t}^{R}, \\
\Gamma_{\varphi \varphi}^{R}=\frac{\sin ^{2} \vartheta}{\Sigma}\left(a^{2} \Sigma \sin ^{2} \vartheta \Gamma_{t t}^{R}-R \Delta\right), \quad \Gamma_{t R}^{t}=\frac{\left(R^{2}+a^{2}\right) \Sigma}{\Delta^{2}} \Gamma_{t t}^{R}, \\
\Gamma_{\varphi R}^{\varphi}=-\frac{1}{\Sigma^{2} \Delta}\left[-R a^{2} \Sigma \sin ^{2} \vartheta \frac{d M}{d R}+a^{2}\left(R^{2}-a^{2} \cos ^{2} \vartheta\right) M(R)\right. \\
\left.+a^{2} \Sigma \cos ^{2} \vartheta M(R)-a^{2} R \cos ^{2} \vartheta\left(2 R^{2}+a^{2} \cos ^{2} \vartheta\right)-R^{4}(R-2 M(R))\right] \\
\Gamma_{R R}^{R}=\frac{1}{\Sigma \Delta}\left(a^{2} R \sin ^{2} \vartheta-\frac{\Sigma^{3}}{\Delta} \Gamma_{t t}^{R}\right), \quad \Gamma_{\vartheta R}^{\vartheta}=\frac{R}{\Sigma}, \quad \Gamma_{\vartheta \vartheta}^{R}=-\frac{R \Delta}{\Sigma}, \\
\Gamma_{t R}^{t}+\Gamma_{\varphi R}^{\varphi}+2 \Gamma_{R R}^{R}+\Gamma_{\vartheta R}^{\vartheta}=\frac{1}{\Sigma \Delta}\left[-\frac{\Sigma^{3}}{\Delta} \Gamma_{t t}^{R}+2 R^{2}(R-2 M(R))+a^{2} R\left(3-\cos ^{2} \vartheta\right)\right],
\end{gathered}
$$

after a tedious manipulation equation (52) becomes

$$
A(R, \vartheta) p_{\vartheta}=B(R, \vartheta) \rho+\frac{\Delta}{\Sigma} \partial_{R} \rho
$$

with

$$
A(R, \vartheta)=\frac{1}{\Sigma}\left(a^{2} \sin ^{2} \vartheta \Gamma_{t t}^{R}+2 a \Gamma_{t \varphi}^{R}+\frac{\Gamma_{\varphi \varphi}^{R}}{\sin ^{2} \vartheta}+\Gamma_{\vartheta \vartheta}^{R}\right)=-\frac{2 R \Delta}{\Sigma^{2}}
$$

and

$$
\begin{aligned}
B(R, \vartheta)=\partial_{R} & \left(\frac{\Delta}{\Sigma}\right)-\frac{\left(R^{2}+a^{2}\right)^{2}}{\Sigma \Delta} \Gamma_{t t}^{R}-\frac{2 a\left(R^{2}+a^{2}\right)}{\Sigma \Delta} \Gamma_{t \varphi}^{R}-\frac{a^{2}}{\Sigma \Delta} \Gamma_{\varphi \varphi}^{R} \\
& +\frac{\Delta}{\Sigma}\left(\Gamma_{t R}^{t}+\Gamma_{\varphi R}^{\varphi}+2 \Gamma_{R R}^{R}+\Gamma_{\vartheta R}^{\vartheta}\right)=\frac{2 R \Delta}{\Sigma^{2}} .
\end{aligned}
$$

Finally, equation (52) reduces to

$$
-p_{\theta}=\rho+\frac{\Sigma}{2 R} \partial_{R} \rho
$$


which coincides with equation (41) in [17]. For the case $\mu=\vartheta$ the conservation equation for the energy-momentum tensor gives rise to the following equation

$$
\partial_{\vartheta} T^{\vartheta \vartheta}+\Gamma_{t t}^{\vartheta} T^{t t}+2 \Gamma_{t \varphi}^{\vartheta} T^{t \varphi}+\Gamma_{\varphi \varphi}^{\vartheta} T^{\varphi \varphi}+\left(\Gamma_{t \vartheta}^{t}+\Gamma_{R \vartheta}^{R}+2 \Gamma_{\vartheta \vartheta}^{\vartheta}+\Gamma_{\vartheta \varphi}^{\varphi}\right) T^{\vartheta \vartheta}+\Gamma_{R R}^{\vartheta} T^{R R}=0
$$

where the Christoffel symbols are given by

$$
\begin{gathered}
\Gamma_{t \varphi}^{\vartheta}=\frac{2 a R}{\Sigma^{3}}\left(R^{2}+a^{2}\right) M(R) \sin \vartheta \cos \vartheta, \quad \Gamma_{t t}^{\vartheta}=-\frac{a}{R^{2}+a^{2}} \Gamma_{t \varphi}^{\vartheta}, \\
\Gamma_{\varphi \varphi}^{\vartheta}=-\frac{\sin \vartheta \cos \vartheta}{\Sigma^{3}}\left[\Sigma^{2} \Delta+2 R\left(R^{2}+a^{2}\right)^{2} M(R)\right], \quad \Gamma_{R R}^{\vartheta}=\frac{a^{2} \sin \vartheta \cos \vartheta}{\Sigma \Delta}, \\
\Gamma_{t \vartheta}^{t}=-\frac{a \Sigma}{R^{2}+a^{2}} \Gamma_{t \varphi}^{\vartheta}, \quad \Gamma_{R \vartheta}^{R}=\Gamma_{\vartheta \vartheta}^{\vartheta}=-\Gamma_{R R}^{\vartheta}, \quad \Gamma_{\varphi \vartheta}^{\varphi}=\frac{\cot \vartheta}{\Sigma}\left(\Sigma^{2}+2 a^{2} R M(R) \sin ^{2} \vartheta\right) \\
\Gamma_{t \vartheta}^{t}+\Gamma_{R \vartheta}^{R}+2 \Gamma_{\vartheta \vartheta}^{\vartheta}+\Gamma_{\vartheta \varphi}^{\varphi}=\frac{\cot \vartheta}{\Sigma}\left(R^{2}+4 a^{2} \cos ^{2} \vartheta-3 a^{2}\right) .
\end{gathered}
$$

After a long computation equation (54) can be written in the more amenable form

$$
\partial_{\vartheta} p_{\vartheta}-\frac{2 a^{2}}{\Sigma} \sin \vartheta \cos \vartheta\left(\rho+p_{\vartheta}\right)=0
$$

Notice that in the limit $a \rightarrow 0$ the above equation reduces to $\partial_{\vartheta} p_{\vartheta}$ as it is expected for the noncommutative inspired Schwarzschild metric. Employing (53) the above equation becomes a partial differential equation for the function $\rho(R, \vartheta)$, namely

$$
\partial_{\vartheta} \rho-\frac{a^{2}}{R} \sin \vartheta \cos \vartheta \partial_{R} \rho+\frac{1}{2 R} \partial_{\vartheta}\left(\Sigma \partial_{R} \rho\right)=0
$$

The above equation can be solved by the method of separation of variables. To this purpose, let $\rho(R, \vartheta)=F_{1}(R) F_{2}(\vartheta)$. Then, we obtain

$$
\dot{F}_{1}=\frac{1}{R F_{1}}\left(2 R \dot{F}_{1}^{2}+F_{1} \dot{F}_{1}\right)
$$

and

$$
\frac{d F_{2}}{d \vartheta}=\frac{2 a^{2} \sin \vartheta \cos \vartheta \dot{F}_{1}}{2 R F_{1}+\Sigma \dot{F}_{1}} F_{2},
$$

where the dot denotes differentiation with respect to the variable $R$. The solution of the first order non linear equation (57) is given by

$$
F_{1}(R)=-\frac{2}{C_{1} R^{2}+C_{2}}
$$

Substituting (59) into (58) we get

$$
\frac{d F_{2}}{d \vartheta}=\frac{2 C_{1} a^{2} \sin \vartheta \cos \vartheta}{a^{2} C_{1} \cos ^{2} \vartheta-2 C_{2}} F_{2},
$$


whose solution is

$$
F_{2}(\vartheta)=-\frac{2 C_{3}}{a^{2} C_{1} \cos 2 \vartheta+a^{2} C_{1}-4 C_{2}} .
$$

On the other side, taking into account that $T_{R R}=-(\Sigma / \Delta) \rho$ and making use of (72) the Einstein field equation $G_{R R}=-8 \pi T_{R R}$ simplifies to

$$
\frac{d M}{d R}=4 \pi \frac{\Sigma^{2}}{R^{2}} \rho
$$

with $\rho(R, \vartheta)=F_{1}(R) F_{2}(\vartheta)$ where the functions $F_{1}(R)$ and $F_{2}(\vartheta)$ are given by (59) and (60) $)$. Since the mass function depends only on the spatial variable $R$, whereas the r.h.s. of the above equation manifests an additional dependence on the angular variable $\vartheta$ which cannot be removed. We have a contradiction and the proof is completed.

\section{CONCLUSIONS}

The present work is in the spirit of [10 14, 17] and deals with the problem of deriving the full set of Einstein field equations assuming an anisotropic energy-momentum tensor, where the energy density is inspired by Noncommutative Geometry and is meant to replace the ring singularity. These equations are written down in an explicit form in (12)-(19) and (36)-(42) if we demand the existence of at least one horizon.. Any candidate solution of the Kerr metric inspired by Noncommutative Geometry has to satisfy these equations. A suggestion for such a solution was made in ([17]) where the metric is written in the KerrSchild form in terms of a null vector $k^{\mu}$. This vector is chosen explicitly on the basis of symmetry arguments such that only one function remains unknown. For this function a differential equation is derived out of the Einstein equations. It is, however, a priori not clear if all Einstein equations are identically satisfied. We did this check and showed in theorem 1, theorem 2 and appendix B that the so-called Kerrr solution can never satisfy the Einstein field equations. In this sense, our results can be seen as complementary to ([17]). We hope that in the future using our set of equations we will be able to examine closer the smeared ring singularity of the Kerr solution inspired by Noncommutative Geometry. 


\section{APPENDIX}

\section{LIST OF THE RELEVANT CHRISTOFFEL SYMBOLS AND TENSOR COMPONENTS}

\section{A. Christoffel symbols}

For the metric (1) the non vanishing Christoffel symbols are

$$
\begin{gathered}
\Gamma^{t}{ }_{t i}=\nu_{, i}-\frac{1}{2} \omega \omega_{, i} e^{2(\psi-\nu)}, \quad \Gamma_{\varphi i}^{t}=\frac{1}{2} \omega_{, i} e^{2(\psi-\nu)}, \\
\Gamma_{t i}^{\varphi}=\omega \nu_{, i}-\frac{1}{2} \omega_{, i}-\omega \psi_{, i}-\frac{1}{2} \omega^{2} \omega_{, i} e^{2(\psi-\nu)}, \quad \Gamma^{\varphi}{ }_{\varphi i}=\psi_{, i}+\frac{1}{2} \omega \omega_{, i} e^{2(\psi-\nu)}, \\
\Gamma_{t t}^{R}=\nu_{, R} e^{2\left(\nu-\mu_{R}\right)}-\left(\omega \omega_{, R}+\omega^{2} \psi_{, R}\right) e^{2\left(\psi-\mu_{R}\right)}, \quad \Gamma^{R}{ }_{t \varphi}=\left(\frac{1}{2} \omega_{, R}+\omega \psi_{, R}\right) e^{2\left(\psi-\mu_{R}\right)}, \\
\Gamma_{\varphi \varphi}^{R}=-\psi_{, R} e^{2\left(\psi-\mu_{R}\right)}, \quad \Gamma_{R R}^{R}=\mu_{R, R}, \quad \Gamma_{R \vartheta}^{R}=\mu_{R, \vartheta}, \quad \Gamma_{\vartheta \vartheta}^{R}=-\mu_{\vartheta, R} e^{2\left(\mu_{\vartheta}-\mu_{R}\right)}, \\
\Gamma_{t t}^{\vartheta}=\nu_{, \vartheta} e^{2\left(\nu-\mu_{\vartheta}\right)}-\left(\omega \omega_{, \vartheta}+\omega^{2} \psi_{, \vartheta}\right) e^{2\left(\psi-\mu_{\vartheta}\right)}, \quad \Gamma_{t \varphi}^{\vartheta}=\left(\frac{1}{2} \omega_{, \vartheta}+\omega \psi_{, \vartheta}\right) e^{2\left(\psi-\mu_{\vartheta}\right)}, \\
\Gamma_{\varphi \varphi}^{\vartheta}=-\psi_{, \vartheta} e^{2\left(\psi-\mu_{\vartheta}\right)}, \quad \Gamma_{R R}^{\vartheta}=-\mu_{R, \vartheta} e^{2\left(\mu_{R}-\mu_{\vartheta}\right)}, \quad \Gamma_{R \vartheta}^{\vartheta}=\mu_{\vartheta, R}, \quad \Gamma_{\vartheta \vartheta}^{\vartheta}=\mu_{\vartheta, \vartheta},
\end{gathered}
$$

where $i=R, \vartheta$.

\section{B. Covariant Riemann tensor components}

The non-vanishing components of the covariant Riemann tensor have been computed according to the formula

$$
R_{\rho \lambda \mu \nu}=\frac{1}{2}\left(g_{\lambda \mu, \rho \nu}-g_{\rho \mu, \lambda \nu}-g_{\lambda \nu, \rho \mu}+g_{\rho \nu, \lambda \mu}\right)+g_{\xi \eta}\left(\Gamma_{\rho \nu}^{\xi} \Gamma_{\lambda \mu}^{\eta}-\Gamma_{\rho \mu}^{\xi} \Gamma_{\lambda \nu}^{\eta}\right) .
$$

For the metric (11) the relevant components of the covariant curvature tensor are

$$
\begin{gathered}
R_{\varphi t \varphi t}=e^{2(\psi+\nu)}\left[-e^{-2 \mu_{R}} \nu_{, R} \psi_{, R}-e^{-2 \mu_{\vartheta}} \nu_{, \vartheta} \psi_{, \vartheta}-\frac{1}{4} e^{2(\psi-\nu)}\left[e^{-2 \mu_{R}}\left(\omega_{, R}\right)^{2}+e^{-2 \mu_{\vartheta}}\left(\omega_{, \vartheta}\right)^{2}\right]\right] \\
R_{R t R t}=e^{2\left(\nu+\mu_{R}\right)}\left[-e^{-\mu_{R}-\nu}\left(e^{\nu-\mu_{R}} \nu_{, R}\right)_{, R}-\nu_{, \vartheta} \mu_{R, \vartheta} e^{-2 \mu_{\vartheta}}+\frac{3}{4}\left(\omega_{, R}\right)^{2} e^{2 \psi-2 \mu_{R}-2 \nu}\right] \\
+\left[\omega \omega_{, R, R}+3 \omega \omega_{, R} \psi_{, R}+\omega^{2} \psi_{, R, R}+\left(\omega \psi_{, R}\right)^{2}-\omega \omega_{, R} \nu_{, R}-\omega \omega_{, R} \mu_{R, R}-\omega^{2} \psi_{, R} \mu_{R, R}\right] e^{2 \psi} \\
+\left[\mu_{R, \vartheta} \omega \omega_{, \vartheta}+\mu_{R, \vartheta} \omega^{2} \psi_{, \vartheta}\right] e^{2 \psi-2 \mu_{\vartheta}+2 \mu_{R}}+\frac{1}{4}\left(\omega \omega_{, R}\right)^{2} e^{4 \psi-2 \nu}
\end{gathered}
$$




$$
\begin{aligned}
& R_{R t R \varphi}=\left[-\frac{1}{2} \omega_{, R, R}-\frac{3}{2} \omega_{, R} \psi_{, R}-\omega \psi_{, R, R}-\omega \psi_{, R}^{2}+\frac{1}{2} \omega_{, R} \nu_{, R}+\frac{1}{2} \omega_{, R} \mu_{R, R}+\omega \psi_{, R} \mu_{R, R}\right] e^{2 \psi} \\
& -\frac{1}{4} \omega \omega_{, R}^{2} e^{4 \psi-2 \nu}-\mu_{R, \vartheta} e^{2 \psi-2 \mu_{\vartheta}+2 \mu_{R}}\left[\frac{1}{2} \omega_{, \vartheta}+\omega \psi_{, \vartheta}\right]=R_{R \varphi R t}, \\
& R_{R \varphi R \varphi}=\left[\psi_{, R, R}+\psi_{, R}^{2}-\mu_{R, R} \psi_{, R}\right] e^{2 \psi}+\frac{1}{4} \omega_{, R}^{2} e^{4 \psi-2 \nu}+\mu_{R, \vartheta} \psi_{, \vartheta} e^{2 \psi+2 \mu_{R}-2 \mu_{\vartheta}}, \\
& R_{\vartheta t \vartheta t}=e^{2\left(\nu+\mu_{\vartheta}\right)}\left[-e^{-\mu_{\vartheta}-\nu}\left(e^{\nu-\mu_{\vartheta}} \nu_{, \vartheta}\right)_{, \vartheta}-\nu_{, R} \mu_{\vartheta, R} e^{-2 \mu_{R}}+\frac{3}{4}\left(\omega_{, \vartheta}\right)^{2} e^{2 \psi-2 \mu_{\vartheta}-2 \nu}\right] \\
& +\left[\omega \omega_{, \vartheta, \vartheta}+3 \omega \omega_{, \vartheta} \psi_{, \vartheta}+\omega^{2} \psi_{, \vartheta, \vartheta}+\left(\omega \psi_{, \vartheta}\right)^{2}-\omega \omega_{, \vartheta} \nu_{, \vartheta}-\omega \omega_{, \vartheta} \mu_{\vartheta, \vartheta}-\omega^{2} \psi_{, \vartheta} \mu_{\vartheta, \vartheta}\right] e^{2 \psi} \\
& +\left[\mu_{\vartheta, R} \omega \omega_{, R}+\mu_{\vartheta, R} \omega^{2} \psi_{, R}\right] e^{2 \psi-2 \mu_{R}+2 \mu_{\vartheta}}+\frac{1}{4}\left(\omega \omega_{, \vartheta}\right)^{2} e^{4 \psi-2 \nu}, \\
& R_{\vartheta t \vartheta \varphi}=\left[-\frac{1}{2} \omega_{, \vartheta, \vartheta}-\frac{3}{2} \omega_{, \vartheta} \psi_{, \vartheta}-\omega \psi_{, \vartheta, \vartheta}-\omega \psi_{, \vartheta}^{2}+\frac{1}{2} \omega_{, \vartheta} \nu_{, \vartheta}+\frac{1}{2} \omega_{, \vartheta} \mu_{\vartheta, \vartheta}+\omega \psi_{, \vartheta} \mu_{\vartheta, \vartheta}\right] e^{2 \psi} \\
& -\frac{1}{4} \omega \omega_{, \vartheta}^{2} e^{4 \psi-2 \nu}-\mu_{\vartheta, R} e^{2 \psi-2 \mu_{R}+2 \mu_{\vartheta}}\left[\frac{1}{2} \omega_{, R}+\omega \psi_{, R}\right]=R_{\vartheta t \vartheta \varphi} \\
& R_{\vartheta \varphi \vartheta \varphi}=\left[\psi_{, \vartheta, \vartheta}+\psi_{, \vartheta}^{2}-\mu_{\vartheta, \vartheta} \psi_{, \vartheta}\right] e^{2 \psi}+\frac{1}{4} \omega_{, \vartheta}^{2} e^{4 \psi-2 \nu}+\mu_{\vartheta, R} \psi_{, R} e^{2 \psi+2 \mu_{\vartheta}-2 \mu_{R}}, \\
& R_{t R t \vartheta}=e^{2 \nu+\mu_{R}+\mu_{\vartheta}}\left[-e^{-\mu_{\vartheta}-\nu}\left(e^{\nu-\mu_{R}} \nu_{, R}\right)_{, \vartheta}+\nu_{, \vartheta} \mu_{\vartheta, R} e^{-\mu_{R}-\mu_{\vartheta}}+\frac{3}{4} \omega_{, R} \omega_{, \vartheta} e^{2 \psi-2 \nu-\mu_{R}-\mu_{\vartheta}}\right] \\
& +\left[\omega \omega_{, R, \vartheta}+\frac{3}{2} \omega \omega_{, R} \psi_{, \vartheta}+\frac{3}{2} \omega \omega_{, \vartheta} \psi_{, R}+\omega^{2} \psi_{, R, \vartheta}+\omega^{2} \psi_{, R} \psi_{, \vartheta}-\frac{1}{2} \omega \omega_{, R} \nu_{, \vartheta}-\frac{1}{2} \omega \omega_{, \vartheta} \nu_{, R}\right. \\
& \left.-\mu_{R, \vartheta}\left(\omega \omega_{, R}+\omega^{2} \psi_{, R}\right)-\mu_{\vartheta, R}\left(\omega \omega_{, \vartheta}+\omega^{2} \psi_{, \vartheta}\right)\right] e^{2 \psi}+\frac{1}{4} \omega^{2} \omega_{, R} \omega_{, \vartheta} e^{4 \psi-2 \nu}, \\
& R_{t R \varphi \vartheta}=\left[-\frac{1}{2} \omega_{, R, \vartheta}-\omega_{, R} \psi_{, \vartheta}-\frac{1}{2} \omega_{, \vartheta} \psi_{, R}-\omega \psi_{, R, \vartheta}-\omega \psi_{, R} \psi_{, \vartheta}+\frac{1}{2} \omega_{, R} \nu_{, \vartheta}\right. \\
& \left.+\mu_{R, \vartheta}\left(\frac{1}{2} \omega_{, R}+\omega \psi_{, R}\right)+\mu_{\vartheta, R}\left(\frac{1}{2} \omega_{, \vartheta}+\omega \psi_{, \vartheta}\right)\right] e^{2 \psi}-\frac{1}{4} \omega \omega_{, R} \omega_{, \vartheta} e^{4 \psi-2 \nu}, \\
& R_{\varphi R t \vartheta}=\left[-\frac{1}{2} \omega_{, R, \vartheta}-\frac{1}{2} \omega_{, R} \psi_{, \vartheta}-\omega_{, \vartheta} \psi_{, R}-\omega \psi_{, R, \vartheta}-\omega \psi_{, R} \psi_{, \vartheta}+\frac{1}{2} \omega_{, \vartheta} \nu_{, R}\right. \\
& \left.+\mu_{R, \vartheta}\left(\frac{1}{2} \omega_{, R}+\omega \psi_{, R}\right)+\mu_{\vartheta, R}\left(\frac{1}{2} \omega_{, \vartheta}+\omega \psi_{, \vartheta}\right)\right] e^{2 \psi}-\frac{1}{4} \omega \omega_{, R} \omega_{, \vartheta} e^{4 \psi-2 \nu}, \\
& R_{\varphi R \varphi \vartheta}=\left[e^{-\psi-\mu_{\vartheta}}\left(e^{\psi-\mu_{R}} \psi_{, R}\right)_{, \vartheta}-\psi, \vartheta \mu_{\vartheta, R} e^{-\mu_{R}-\mu_{\vartheta}}+\frac{1}{4} \omega_{, R} \omega_{, \vartheta} e^{2 \psi-2 \nu-\mu_{R}-\mu_{\vartheta}}\right] e^{2 \psi+\mu_{R}+\mu_{\vartheta}}, \\
& R_{\vartheta R \vartheta R}=\left[e^{-\mu_{R}-\mu_{\vartheta}}\left(e^{\mu_{R}-\mu_{\vartheta}} \mu_{R, \vartheta}\right)_{, \vartheta}+e^{-\mu_{R}-\mu_{\vartheta}}\left(e^{\mu_{\vartheta}-\mu_{R}} \mu_{\vartheta, R}\right)_{, R}\right] e^{2 \mu_{R}+2 \mu_{\vartheta}} .
\end{aligned}
$$




\section{Covariant Riemann tensor components in a non coordinate basis}

Starting with the metric (1) we can take the basis one-forms [16, 18]

$$
e^{(t)}=e^{\nu} d t, \quad e^{(\varphi)}=-\omega e^{\psi} d t+e^{\psi} d \varphi, \quad e^{(R)}=e^{\mu_{R}} d R, \quad e^{(\vartheta)}=e^{\mu_{\vartheta}} d \vartheta
$$

where we enclosed tetrad indices in a parenthesis in order to distinguish them from tensor indices. The vierbein associated to the above choice of the basis one-forms is

$$
e_{(t)}=e^{-\nu} \partial_{t}+\omega e^{-\nu} \partial_{\varphi}, \quad e_{(\varphi)}=e^{-\psi} \partial_{\varphi}, \quad e_{(2)}=e^{-\mu_{2}} \partial_{2}, \quad e_{(3)}=e^{-\mu_{3}} \partial_{3}
$$

Moreover, the chosen tetrad satisfies the relation

$$
e_{(a)}^{\mu} e_{(b)}{ }^{\nu} g_{\mu \nu}=\eta_{(a)(b)}, \quad \eta_{(a)(b)}=\operatorname{diag}(1,-1,-1,-1) .
$$

The tetrad components of the covariant Riemann tensor are given by

$$
R_{(a)(b)(c)(d)}=R_{\mu \nu \rho \lambda} e_{(a)}^{\mu} e_{(b)}^{\nu} e_{(c)}^{\rho} e_{(d)}^{\lambda} .
$$

The non-vanishing components of the covariant Riemann tensor are

$$
\begin{aligned}
& R_{(\varphi)(t)(\varphi)(t)}=\left[e_{(t)}{ }^{t} e_{(\varphi)}^{\varphi}\right]^{2} R_{\varphi t \varphi t} \\
& =-e^{-2 \mu_{R}} \nu_{, R} \psi_{, R}-e^{-2 \mu_{\vartheta}} \nu_{, \vartheta} \psi_{, \vartheta}-\frac{1}{4} e^{2(\psi-\nu)}\left[e^{-2 \mu_{R}}\left(\omega_{, R}\right)^{2}+e^{-2 \mu_{\vartheta}}\left(\omega_{, \vartheta}\right)^{2}\right], \\
& R_{(R)(t)(R)(t)}=\left[e_{(t)}{ }^{t} e_{(R)}{ }^{R}\right]^{2} R_{R t R t}+2\left[e_{(R)}{ }^{R}\right]^{2} e_{(t)}{ }^{t} e_{(t)}{ }^{\varphi} R_{R t R \varphi}+\left[e_{(t)} e_{(R)}{ }^{R}\right]^{2} R_{R \varphi R \varphi} \\
& =-e^{-\mu_{R}-\nu}\left(e^{\nu-\mu_{R}} \nu_{, R}\right)_{, R}-\nu_{, \vartheta} \mu_{R, \vartheta} e^{-2 \mu_{\vartheta}}+\frac{3}{4}\left(\omega_{, R}\right)^{2} e^{2 \psi-2 \mu_{R}-2 \nu}, \\
& R_{(\vartheta)(t)(\vartheta)(t)}=\left[e_{(t)}^{t} e_{(\vartheta)}{ }^{\vartheta}\right]^{2} R_{\vartheta t \vartheta t}+2\left[e_{(\vartheta)}{ }^{\vartheta}\right]^{2} e_{(t)}{ }^{t} e_{(t)}{ }^{\varphi} R_{\vartheta t \vartheta \varphi}+\left[e_{(t)} e_{(\vartheta)}\right]^{2} R_{\vartheta \varphi \vartheta \varphi} \\
& =-e^{-\mu_{\vartheta}-\nu}\left(e^{\nu-\mu_{\vartheta}} \nu_{, \vartheta}\right)_{, \vartheta}-\nu_{, R} \mu_{\vartheta, R} e^{-2 \mu_{R}}+\frac{3}{4}\left(\omega_{, \vartheta}\right)^{2} e^{2 \psi-2 \mu_{\vartheta}-2 \nu}, \\
& R_{(R)(t)(R)(\varphi)}=\left[e_{(R)}{ }^{R}\right]^{2} e_{(t)}{ }^{t} e_{(\varphi)}{ }^{\varphi} R_{R t R \varphi}+\left[e_{(R)}{ }^{R}\right]^{2} e_{(t)}{ }^{\varphi} e_{(\varphi)}{ }^{\varphi} R_{R \varphi R \varphi} \\
& =-\omega_{, R}\left(\psi_{, R}-\frac{1}{2} \nu_{, R}\right) e^{\psi-\nu-2 \mu_{R}}-\frac{1}{2} e^{-\mu_{R}-\nu}\left(e^{\psi-\mu_{R}} \omega_{, R}\right)_{, R}-\frac{1}{2} \omega_{, \vartheta} \mu_{R, \vartheta} e^{\psi-\nu-2 \mu_{\vartheta}}, \\
& R_{(\vartheta)(t)(\vartheta)(\varphi)}=\left[e_{(\vartheta)}^{\vartheta}\right]^{2} e_{(t)}^{t} e_{(\varphi)}^{\varphi} R_{\vartheta t \vartheta \varphi}+\left[e_{(\vartheta)}\right]^{2} e_{(t)} e_{(\varphi)}^{\varphi} R_{\vartheta \varphi \vartheta \varphi} \\
& =-\omega_{, \vartheta}\left(\psi_{, \vartheta}-\frac{1}{2} \nu_{, \vartheta}\right) e^{\psi-\nu-2 \mu_{\vartheta}}-\frac{1}{2} e^{-\mu_{\vartheta}-\nu}\left(e^{\psi-\mu_{\vartheta}} \omega_{, \vartheta}\right)_{, \vartheta}-\frac{1}{2} \omega_{, R} \mu_{\vartheta, R} e^{\psi-\nu-2 \mu_{R}} \text {, } \\
& R_{(R)(\varphi)(R)(\varphi)}=\left[e_{(R)} e_{(\varphi)}^{\varphi}\right]^{2} R_{R \varphi R \varphi}
\end{aligned}
$$




$$
\begin{gathered}
=e^{-\psi-\mu_{R}}\left(e^{\psi-\mu_{R}} \psi_{, R}\right)_{, R}+\mu_{R, \vartheta} \psi_{, \vartheta} e^{-2 \mu_{\vartheta}}+\frac{1}{4} \omega_{, R}^{2} e^{2 \psi-2 \mu_{R}-2 \nu}, \\
R_{(\vartheta)(\varphi)(\vartheta)(\varphi)}=\left[e_{(\vartheta)} e_{(\varphi)}\right]^{2} R_{\vartheta \varphi \vartheta \varphi} \\
=e^{-\psi-\mu_{\vartheta}}\left(e^{\psi-\mu_{\vartheta}} \psi_{, \vartheta}\right)_{, \vartheta}+\mu_{\vartheta, R} \psi_{, R} e^{-2 \mu_{R}}+\frac{1}{4} \omega_{, \vartheta}^{2} e^{2 \psi-2 \mu_{\vartheta}-2 \nu}, \\
R_{(t)(R)(t)(\vartheta)}=\left[e_{(t)}{ }^{t}\right]^{2} e_{(R)}{ }^{R} e_{(\vartheta)}{ }^{\vartheta} R_{t R t \vartheta}+\left(R_{t R \varphi \vartheta}+R_{\varphi R t \vartheta}\right) e_{(t)}{ }^{t} e_{(t)} e_{(R)} e_{(\vartheta)} \vartheta \\
\left.+\left[e_{(t)}\right]^{\varphi}\right]_{(R)} e_{(\vartheta)} e_{(\vartheta} R_{\varphi R \varphi \vartheta} \\
=-e^{-\nu-\mu_{\vartheta}}\left(e^{\nu-\mu_{R}} \nu_{, R}\right)_{, \vartheta}+\nu_{, \vartheta} \mu_{\vartheta, R} e^{-\mu_{R}-\mu_{\vartheta}}+\frac{3}{4} \omega_{, R} \omega_{, \vartheta} e^{2 \psi-2 \nu-\mu_{R}-\mu_{\vartheta}}, \\
R_{(\varphi)(R)(\varphi)(\vartheta)}=\left[e_{(\varphi)}\right]^{2} e_{(R)}{ }^{R} e_{(\vartheta)}{ }^{\vartheta} R_{\varphi R \varphi \vartheta} \\
R_{(\vartheta)(R)(\vartheta)(R)}=\left[e^{-\psi-\mu_{\vartheta}}\left(e^{\psi-\mu_{R}} \psi_{, R}\right)_{, \vartheta}-\psi_{, \vartheta} \mu_{\vartheta, R} e^{-\mu_{R}-\mu_{\vartheta}}+\frac{1}{4} \omega_{, R} \omega_{, \vartheta} e^{2 \psi-2 \nu-\mu_{R}-\mu_{\vartheta}},\right. \\
R_{\vartheta R \vartheta R}=e^{-\mu_{R}-\mu_{\vartheta}}\left[\left(e^{\mu_{R}-\mu_{\vartheta}} \mu_{R, \vartheta}\right)_{, \vartheta}+\left(e^{\mu_{\vartheta}-\mu_{R}} \mu_{\vartheta, R}\right)_{, R}\right] .
\end{gathered}
$$

\section{Ricci tensor components}

The tetrad components of the Ricci tensor have been computed according to the formula

$$
R_{(a)(b)}=\eta^{(c)(d)} R_{(c)(a)(d)(b)} .
$$

The non-vanishing components are

$$
\begin{gathered}
R_{(t)(t)}=-R_{(\varphi)(t)(\varphi)(t)}-R_{(R)(t)(R)(t)}-R_{(\vartheta)(t)(\vartheta)(t)} \\
=e^{-2 \mu_{R}}\left[\nu_{, R, R}+\nu_{, R}\left(\psi+\nu-\mu_{R}+\mu_{\vartheta}\right)_{, R}\right]+e^{-2 \mu_{\vartheta}}\left[\nu_{, \vartheta, \vartheta}+\nu_{, \vartheta}\left(\psi+\nu+\mu_{R}-\mu_{\vartheta}\right)_{, \vartheta}\right] \\
-\frac{1}{2} e^{2(\psi-\nu)}\left[\left(\omega_{, R}\right)^{2} e^{-2 \mu_{R}}+\left(\omega_{, \vartheta}\right)^{2} e^{-2 \mu_{\vartheta}}\right] \\
R_{(t)(\varphi)}=-R_{(R)(t)(R)(\varphi)}-R_{(\vartheta)(t)(\vartheta)(\varphi)} \\
=\frac{1}{2} e^{-2 \psi-\mu_{R}-\mu_{\vartheta}}\left[\left(\omega_{, R} e^{3 \psi-\nu-\mu_{R}+\mu_{\vartheta}}\right)_{, R}+\left(\omega_{, \vartheta} e^{3 \psi-\nu-\mu_{\vartheta}+\mu_{R}}\right)_{, \vartheta}\right] \\
R_{(\varphi)(\varphi)}=R_{(\varphi)(t)(\varphi)(t)}-R_{(R)(\varphi)(R)(\varphi)}-R_{(\vartheta)(\varphi)(\vartheta)(\varphi)} \\
-\frac{1}{2} e^{2(\psi-\nu)}\left[\left(\omega_{, R}\right)^{2} e^{-2 \mu_{R}}+\left(\omega_{, \vartheta}\right)^{2} e^{-2 \mu_{\vartheta}}\right] \\
R_{(R)(\vartheta)}=R_{(t)(R)(t)(\vartheta)}-R_{(\varphi)(R)(\varphi)(\vartheta)}=-e^{-\mu_{R}-\mu_{\vartheta}} \cdot \\
\left.(\psi+\nu)_{, R, \vartheta}-(\psi+\nu)_{, R} \mu_{R, \vartheta}-(\psi+\nu)_{, \vartheta} \mu_{\vartheta, R}+\psi_{, R} \psi_{, \vartheta}+\nu_{, R} \nu_{, \vartheta}-\frac{1}{2} \omega_{, R} \omega_{, \vartheta} e^{2(\psi-\nu)}\right]
\end{gathered}
$$




$$
\begin{gathered}
R_{(R)(R)}=-R_{(t)(R)(t)(R)}-R_{(\varphi)(R)(\varphi)(R)}-R_{(\vartheta)(R)(\vartheta)(R)} \\
=-e^{-\mu_{R}-\nu}\left(e^{\nu-\mu_{R}} \nu_{, R}\right)_{, R}-e^{-\mu_{R}-\psi}\left(e^{\psi-\mu_{R}} \psi_{, R}\right)_{, R}-e^{-2 \mu_{\vartheta}}(\psi+\nu)_{, \vartheta} \mu_{R, \vartheta} \\
-e^{-\mu_{R}-\mu_{\vartheta}}\left[\left(e^{\mu_{R}-\mu_{\vartheta}} \mu_{R, \vartheta}\right)_{, \vartheta}+\left(e^{\mu_{\vartheta}-\mu_{R}} \mu_{\vartheta, R}\right)_{, R}\right]+\frac{1}{2}\left(\omega_{, R}\right)^{2} e^{2 \psi-2 \mu_{R}-2 \nu} \\
R_{(\vartheta)(\vartheta)}=-R_{(t)(\vartheta)(t)(\vartheta)}-R_{(\varphi)(\vartheta)(\varphi)(\vartheta)}-R_{(R)(\vartheta)(R)(\vartheta)} \\
=-e^{-\mu_{\vartheta}-\nu}\left(e^{\nu-\mu_{\vartheta}} \nu_{, \vartheta}\right)_{, \vartheta}-e^{-\mu_{\vartheta}-\psi}\left(e^{\psi-\mu_{\vartheta}} \psi_{, \vartheta}\right)_{, \vartheta}-e^{-2 \mu_{R}}(\psi+\nu)_{, R} \mu_{\vartheta, R} \\
-e^{-\mu_{R}-\mu_{\vartheta}}\left[\left(e^{\mu_{R}-\mu_{\vartheta}} \mu_{R, \vartheta}\right)_{, \vartheta}+\left(e^{\mu_{\vartheta}-\mu_{R}} \mu_{\vartheta, R}\right)_{, R}\right]+\frac{1}{2}\left(\omega_{, \vartheta}\right)^{2} e^{2 \psi-2 \mu_{\vartheta}-2 \nu} .
\end{gathered}
$$

Finally, the components $G_{(R)(R)}$ and $G_{(\vartheta)(\vartheta)}$ of the Einstein tensor are computed to be

$$
\begin{gathered}
G_{(R)(R)}=e^{-2 \mu_{R}}\left[\nu_{, R}\left(\psi+\mu_{\vartheta}\right)_{, R}+\psi_{, R} \mu_{\vartheta, R}\right]+\frac{1}{4} e^{2 \psi-2 \nu}\left[\left(\omega_{, R}\right)^{2} e^{-2 \mu_{R}}-\left(\omega_{, \vartheta}\right)^{2} e^{-2 \mu_{\vartheta}}\right] \\
+e^{-2 \mu_{\vartheta}}\left[(\psi+\nu)_{, \vartheta, \vartheta}+(\psi+\nu)_{, \vartheta}\left(\nu-\mu_{\vartheta}\right)_{, \vartheta}+\psi_{, \vartheta} \psi_{, \vartheta}\right] \\
G_{(\vartheta)(\vartheta)}=e^{-2 \mu_{R}}\left[(\psi+\nu)_{, R, R}+(\psi+\nu)_{, R}\left(\nu-\mu_{R}\right)_{, R}+\psi_{, R} \psi_{, R}\right] \\
+e^{-2 \mu_{\vartheta}}\left[\nu_{, \vartheta}\left(\psi+\mu_{R}\right)_{, \vartheta}+\psi_{, \vartheta} \mu_{R, \vartheta}\right]-\frac{1}{4} e^{2 \psi-2 \nu}\left[\left(\omega_{, R}\right)^{2} e^{-2 \mu_{R}}-\left(\omega_{, \vartheta}\right)^{2} e^{-2 \mu_{\vartheta}}\right] .
\end{gathered}
$$

\section{KERRR METRIC REVISITED}

We show that the so-called Kerrr metric [17]

$$
\begin{gathered}
d s^{2}=\left(1-\frac{2 R M(R)}{\Sigma}\right) d t^{2}+\frac{4 a R M(R)}{\Sigma} \sin ^{2} \vartheta d t d \varphi-\frac{\Sigma}{\Delta} d R^{2}-\Sigma d \vartheta^{2} \\
-\frac{\sin ^{2} \vartheta}{\Sigma}\left[\left(R^{2}+a^{2}\right)^{2}-a^{2} \sin ^{2} \vartheta \Delta\right] d \varphi^{2}
\end{gathered}
$$

with

$$
\Sigma=R^{2}+a^{2} \cos ^{2} \vartheta, \quad \Delta=R^{2}-2 R M(R)+a^{2}
$$

does not satisfy Einstein field equations $G_{\mu \nu}=-8 \pi T_{\mu \nu}$ with energy-momentum tensor

$$
\begin{gathered}
T_{\nu}^{\mu}=\left(\rho+p_{\vartheta}\right)\left(u^{\mu} u_{\nu}-\ell^{\mu} \ell_{\nu}\right)-p_{\vartheta} \delta_{\nu}^{\mu}, \\
u^{\mu}=\sqrt{-g^{R R}}\left(\delta_{t}^{\mu}+\Omega \delta_{\mu}^{\varphi}\right), \quad \frac{u^{\varphi}}{u^{t}}=\Omega=\frac{a}{R^{2}+a^{2}}, \quad \ell^{\mu}=-\frac{1}{\sqrt{-g_{R R}}} \delta_{R}^{\mu}
\end{gathered}
$$

and $\rho$ given by

$$
\rho(R, \vartheta)=\frac{R^{4}}{\Sigma^{2}} \rho_{G}(R), \quad \rho_{G}(R)=\frac{M}{8 \pi^{3 / 2} \ell_{0}^{3}} e^{-R^{2} / 4 \ell_{0}^{2}}, \quad \rho+p_{\vartheta}=-\frac{\Sigma}{2 R} \partial_{R} \rho .
$$


In contrast to the theorems 1 and 2 we use here the four-velocities as given in [17]. Notice that $\rho_{G}$ is a function depending uniquely on the spatial variable $R$. For the present purpose it is sufficient to consider the Einstein field equations

$$
G_{t t}=-8 \pi T_{t t}, \quad G_{R R}=-8 \pi T_{R R}
$$

By means of the software package Maple 12 we found that the components $G_{t t}$ and $G_{R R}$ of the Einstein tensor are given by

$$
\begin{gathered}
G_{t t}=\frac{1}{\Sigma^{3}}\left[a^{2} R \Sigma \sin ^{2} \vartheta \frac{d^{2} M}{d R^{2}}-2\left(R^{2} \Delta-a^{4} \sin ^{2} \vartheta \cos ^{2} \vartheta\right) \frac{d M}{d R}\right], \\
G_{R R}=\frac{2 R^{2}}{\Sigma \Delta} \frac{d M}{d R} .
\end{gathered}
$$

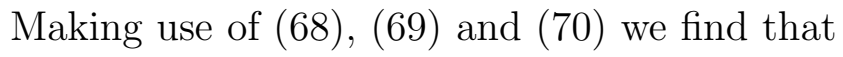

$$
\begin{gathered}
T_{t t}=-\frac{\Delta^{3}}{2 R\left(R^{2}+a^{2}\right)^{2}} \partial_{R}\left(\frac{R^{4}}{\Sigma^{2}} \rho_{G}(R)\right) \\
+\left(1-\frac{2 R M(R)}{\Sigma}\right)\left[\frac{R^{4}}{\Sigma^{2}} \rho_{G}(R)+\frac{\Sigma}{2 R} \partial_{R}\left(\frac{R^{4}}{\Sigma^{2}} \rho_{G}(R)\right)\right], \quad T_{R R}=-\frac{R^{4}}{\Sigma \Delta} \rho_{G}(R) .
\end{gathered}
$$

As in [17] from the equation $G_{R R}=-8 \pi T_{R R}$ we obtain the following result for the mass function $M(R)$, namely

$$
M(r)=4 \pi \int_{0}^{R} d x x^{2} \rho_{G}(x) .
$$

Concerning the equation $G_{t t}=-8 \pi T_{t t}$ it is convenient to rewrite the component $T_{t t}$ as follows

$$
\begin{gathered}
T_{t t}=-\frac{\Delta^{3}}{2 R\left(R^{2}+a^{2}\right)^{2}}\left(4 \frac{R^{2}}{\Sigma^{2}} \rho_{G}(R)-4 \frac{R^{4}}{\Sigma^{3}} \rho_{G}(R)+\frac{R^{3}}{\Sigma^{2}} \frac{d \rho_{G}}{d R}\right)+ \\
\left(1-\frac{2 R M(R)}{\Sigma}\right)\left(2 \frac{R^{2}}{\Sigma} \rho_{G}(R)-\frac{R^{4}}{\Sigma^{2}} \rho_{G}(R)+\frac{R^{3}}{2 \Sigma} \frac{d \rho_{G}}{d R}\right) .
\end{gathered}
$$

Equating (171) to $-8 \pi(74)$ and multiplying by $\Sigma^{3}$ we obtain

$$
\begin{gathered}
a^{2} R \Sigma \sin ^{2} \vartheta \frac{d^{2} M}{d R^{2}}-2\left(R^{2} \Delta-a^{4} \sin ^{2} \vartheta \cos ^{2} \vartheta\right) \frac{d M}{d R} \\
=8 \pi R^{2}\left[\frac{2 \Delta^{3}\left(\Sigma-R^{2}\right)}{\left(R^{2}+a^{2}\right)^{2}}-(\Sigma-2 R M(R))\left(2 \Sigma-R^{2}\right)\right] \rho_{G}(R)+ \\
4 \pi R^{3} \Sigma\left[\frac{\Delta^{3}}{\left(R^{2}+a^{2}\right)^{2}}-(\Sigma-2 R M(R))\right] \frac{d \rho_{G}}{d R} .
\end{gathered}
$$


In order to further simplify the above expression we shall apply (73) to the l.h.s. of the above equation. Therefore, taking into account that

$$
\begin{gathered}
a^{2} R \Sigma \sin ^{2} \vartheta \frac{d^{2} M}{d R^{2}}-2\left(R^{2} \Delta-a^{4} \sin ^{2} \vartheta \cos ^{2} \vartheta\right) \frac{d M}{d R} \\
=8 \pi R^{2}\left[2 a^{4} \sin ^{2} \vartheta \cos ^{2} \vartheta-R^{2}(\Sigma-2 R M(R))\right] \rho_{G}(R)+4 \pi a^{2} R^{3} \Sigma \sin ^{2} \vartheta \frac{d \rho_{G}}{d R},
\end{gathered}
$$

we finally obtain the equation

$$
\begin{gathered}
8 \pi R^{2}\left[\frac{2 \Delta^{3}\left(\Sigma-R^{2}\right)}{\left(R^{2}+a^{2}\right)^{2}}-(\Sigma-2 R M(R))\left(2 \Sigma-R^{2}\right)+R^{2}(\Sigma-2 R M(R))\right. \\
\left.-2 a^{4} \sin ^{2} \vartheta \cos ^{2} \vartheta\right] \rho_{G}(R)+4 \pi R^{2}\left[\frac{\Delta^{3} \Sigma}{\left(R^{2}+a^{2}\right)^{2}}\right. \\
\left.-\Sigma(\Sigma-2 R M(R))-a^{2} \Sigma \sin ^{2} \vartheta\right] \frac{d \rho_{G}}{d R}=0 .
\end{gathered}
$$

Observing that

$$
-(\Sigma-2 R M(R))\left(2 \Sigma-R^{2}\right)+R^{2}(\Sigma-2 R M(R))-2 a^{4} \sin ^{2} \vartheta \cos ^{2} \vartheta=-2 a^{2} \Delta \cos ^{2} \vartheta
$$

and

$$
-(\Sigma-2 R M(R))\left(2 \Sigma-R^{2}\right)-a^{2} \Sigma \sin ^{2} \vartheta=-\Sigma \Delta
$$

equation (75) simplifies as follows

$$
4\left[\frac{\Delta^{2}\left(\Sigma-R^{2}\right)}{\left(R^{2}+a^{2}\right)^{2}}-a^{2} \cos ^{2} \vartheta\right] \rho_{G}(R)+R \Sigma\left[\frac{\Delta^{2}}{\left(R^{2}+a^{2}\right)^{2}}-1\right] \frac{d \rho_{G}}{d R}=0 .
$$

Since

$$
\frac{\Delta^{2}\left(\Sigma-R^{2}\right)}{\left(R^{2}+a^{2}\right)^{2}}-a^{2} \cos ^{2} \vartheta=a^{2} \cos ^{2} \vartheta\left[\frac{\Delta^{2}}{\left(R^{2}+a^{2}\right)^{2}}-1\right]
$$

equation (76) takes the final form

$$
\frac{d \rho_{G}}{d R}+\frac{4 a^{2} \cos ^{2} \vartheta}{R \Sigma} \rho_{G}(R)=0 .
$$

Hence, we have a contradiction since it has been assumed that $\rho_{G}$ depends uniquely on the variable $R$. 


\section{CHOICES OF THE ENERGY DENSITIES}

Throughout the paper we have kept the density $\rho_{\theta}$ as a free function. However, a few comments regarding the choice are in order. The authors of ([17]) advocate the following form

$$
\rho_{\theta}(R, \vartheta)=\frac{M}{8 \pi^{3 / 2} \ell_{0}^{3 / 2}} \frac{R^{2}}{\Sigma} e^{-R^{2} / 4 \ell_{0}^{2}},
$$

where $\Sigma=R^{2}+a^{2} \cos ^{2} \vartheta, a$ is the angular momentum of the black hole per unit mass and $R, \vartheta, \varphi$ are Boyer-Lindquist coordinates. According to the prescription outlined in the introduction it appears to us that a more sophisticated choice might also be possible. Consider the classical ring singularity

$$
\rho_{c l}(x, y, z)=M \delta\left(x^{2}+y^{2}-a^{2}\right) \delta(z)
$$

Then, every $\delta$-distribution must be replaced by a separate Gaussian distribution. This would give us in cylindrical coordinates a density proportional to

$$
\rho(\xi, z) \propto e^{-(\xi-a)^{2} / 4 \theta} e^{-z^{2} / 4 \theta}
$$

where $\xi=\sqrt{x^{2}+y^{2}}$. Of course, this equation is written in cylindrical coordinates and should be rewritten in Boyer-Lindquist coordinates. 
[1] Madore J 2000 An introduction to noncommutative geometry in "Geometry and Quantum Physics", Lectures Notes in Physics Vol. 543, Springer-Verlag; Chamseddine A H, Felder G and Fröhlich J 2005 Gravity in non-commutative geometry' Commun. Math. Phys. 155, 1993; Connes A 1995 Noncommutative geometry and reality, J. Math. Phys. 36, 6194

[2] Alvarez Gaume L and Wadia S R 2001 Gauge theory on a quantum phase space Phys. Lett. B 501, 319; Alvarez Gaume L and Barbon J L F 2001 Nonlinear vacuum phenomena in noncommutative QED Int. J. Mod. Phys. A 16, 1123

[3] Weyl H 1927 Quantenmechanik und Gruppentheorie Z. Phys. 46 1; Wigner E P 1932 On the Quantum Correction For Thermodynamic Equilibrium Phys. Rev. 40, 749; Moyal G E 1949 Quantum mechanics as a statistical theory Proc. Camb. Phil. Soc. 45, 99

[4] Peet A W and Polchinski J 1999 UV-IR relations in AdS dynamics Phys. Rev. D 59, 065011

[5] Smailagic A and Spallucci E 2003 UV divergence-free QFT on noncommutative plane J. Phys. A: Math. Gen. 36, 517

[6] Glauber R J 1963 Coherent and Incoherent States of the Radiation Field Phys. Rev. 131, 2766

[7] Snyder H S 1949 Quantized Space-Time Phys. Rev. 71, 38

[8] Smailagic A and Spallucci E 2003 Feynman Path Integral on the Noncommutative Plane J. Phys. A: Math. Gen. 36, 467

[9] Banerjee R, Majhi B R and Samanta S 2008 Noncommutative Black Hole Thermodynamics Phys. Rev. D 77, 124035; Banerjee R, Majhi B R and Modak S K 2009 Noncommutative Schwarzschild Black Hole and Area Law Class. Quant. Grav. 26, 085010; Banerjee R, Gangopadhyay S and Modak S K 2010 Voros product, Noncommutative Schwarzschild Black Hole and Corrected Area Law, Phys. Lett. B 686, 181

[10] Nicolini P and Spallucci E 2010 Noncommutative geometry inspired dirty black holes Class. Quant. Grav. 27, 015010

[11] Nicolini P 2009 Noncommutative Black Holes, The Final Appeal To Quantum Gravity: A Review Int. J. Mod. Phys. A 24, 1229

[12] Spallucci E, Smailagic A and Nicolini P 2009 Non-commutative geometry inspired higherdimensional charged, black holes Phys. Lett. B 670, 449 
[13] Ansoldi S, Nicolini P, Smailagic A and Spallucci E 2007 Noncommutative geometry inspired charged black holes Phys. Lett. B 645, 261

[14] Nicolini P, Smailagic A and Spallucci E 2006 Noncommutative geometry inspired Schwarzschild black hole Phys. Lett. B 632, 547

[15] Arraut I, Batic D and Nowakowski M 2010 Maximal extension of the Schwarzschild spacetime inspired by noncommutative geometry J. Math. Phys. 51, 022503; Arraut I, Batic D and Nowakowski M 2009 A noncommutative model for a mini black hole Class. Quantum Grav. 26, 245006

[16] Chandrasekhar S and Friedman J L 1972 On the stability of axisymmetric systems to axisymmetric perturbations in General Relativity. I Astrophys. J. 175, 379

[17] Smailagic A and Spallucci E 2010 "Kerrr" black hole: the Lord of the String Phys. Lett. B 688,82

[18] Chandrasekhar S 1983 The Mathematical Theory of Black Holes, Clarendon Press

[19] Boyer R H and Lindquist R W 1967 Maximal Analytic Extension of the Kerr Metric J. Math. Phys 8, 265

[20] Plebanski J and Krasinski A 2006 An Introduction to General Relativity and Cosmology, Cambridge University Press 